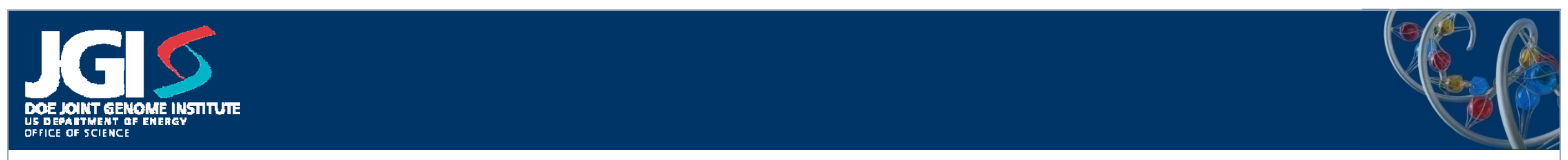

\title{
Ergonomics in DNA Sequencing Standing Down to Ergonomics
}

Presented at the COEH 2009 Summer Institute by Christine Naca July 30, 2009
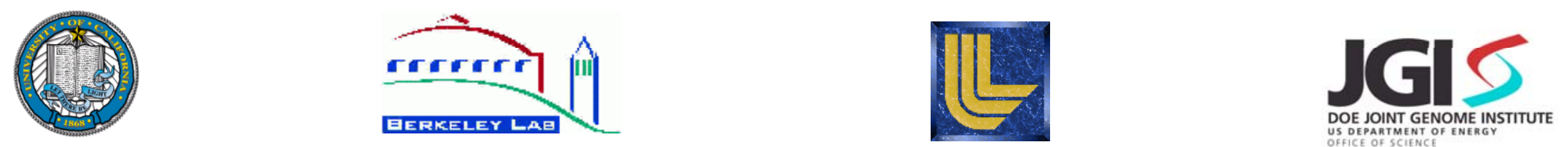


\section{Overview}

- What We Do: Introduction

- How We Work: Ergonomics Challenges in Biotechnology

- Ergonomics Stand Down

- Questions 


POE JGI

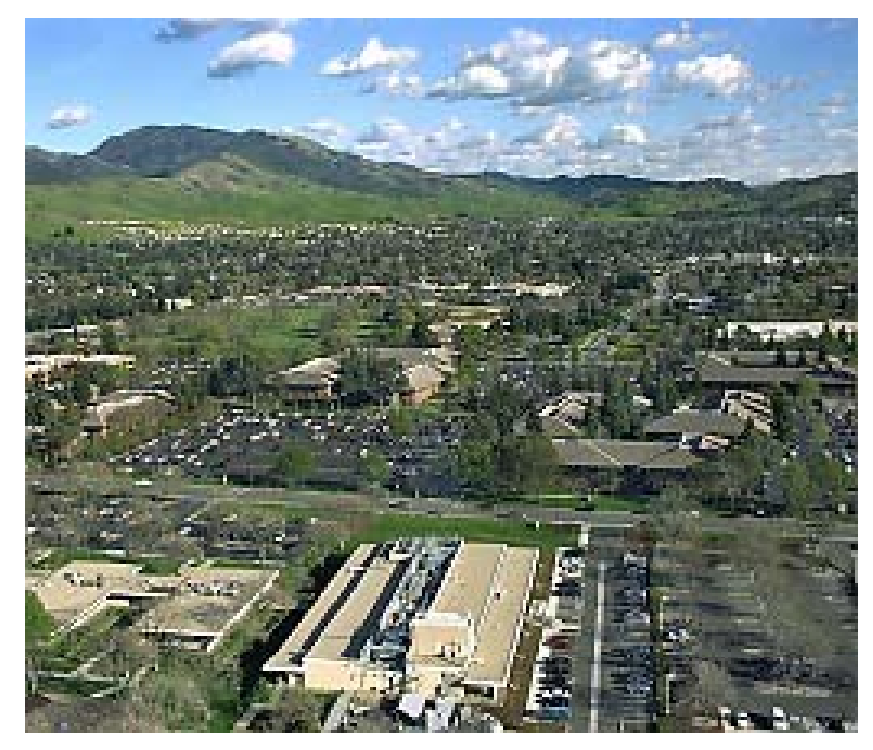

Mission:
- Walnut Creek, CA-located PGF opened in 1999

- 250 employees

DOE JGI, Serving as a genomic user facility in support of the DOE missions: bioenergy, carbon cycling, and bioremediation. 


The Human Genome
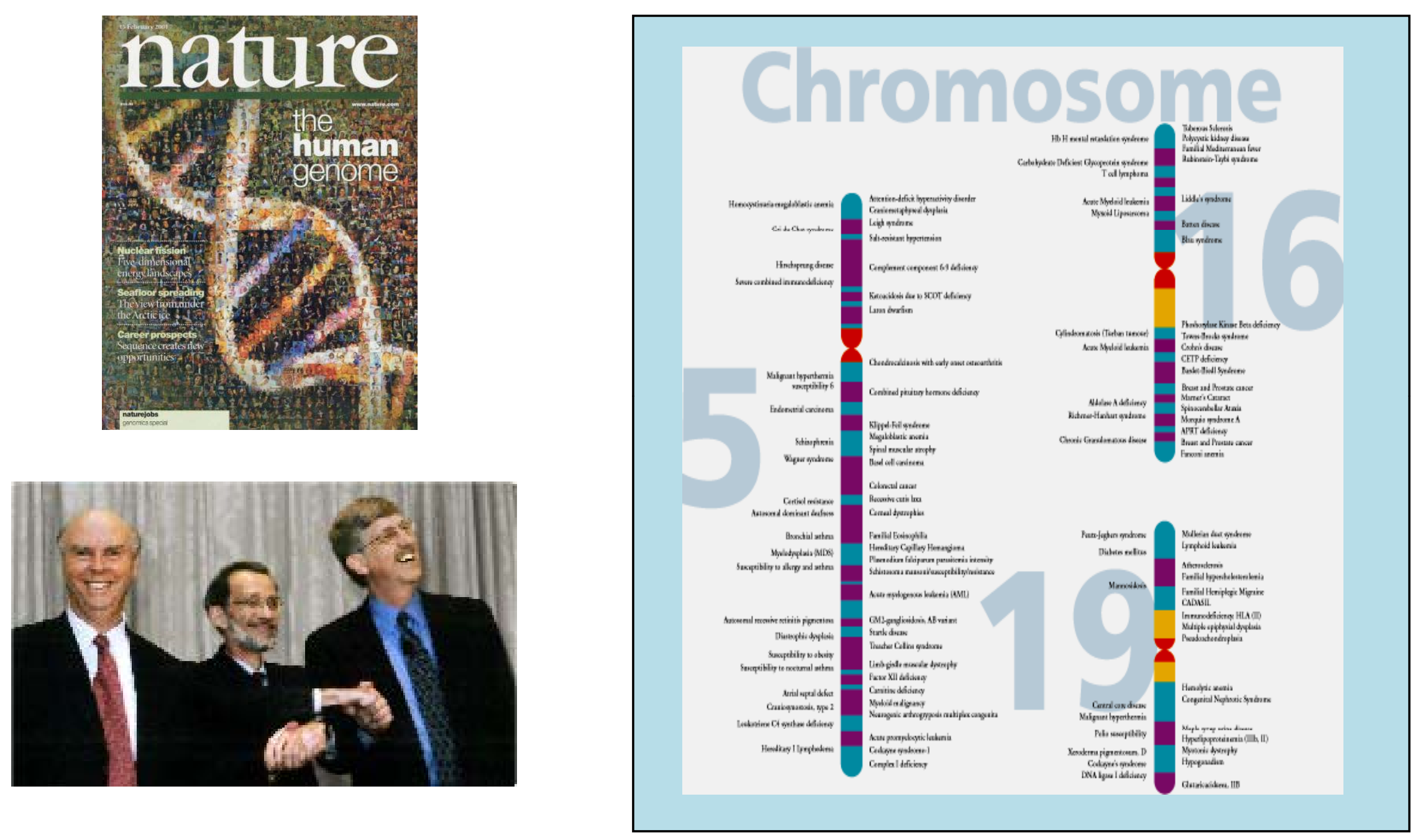


\section{(C) \\ DOE Mission Relevance}

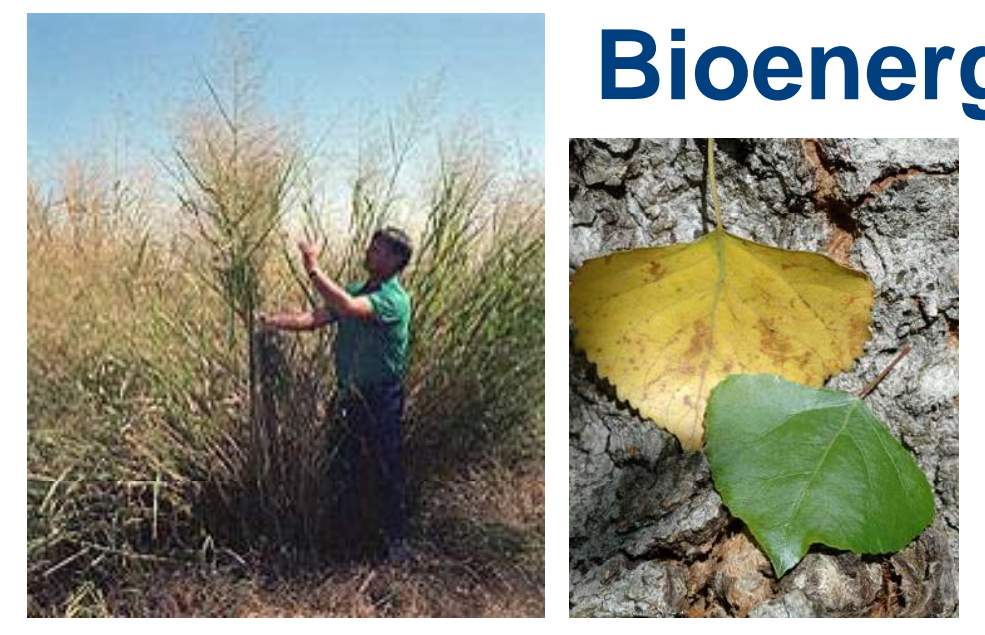

\section{Carbon Cycling}
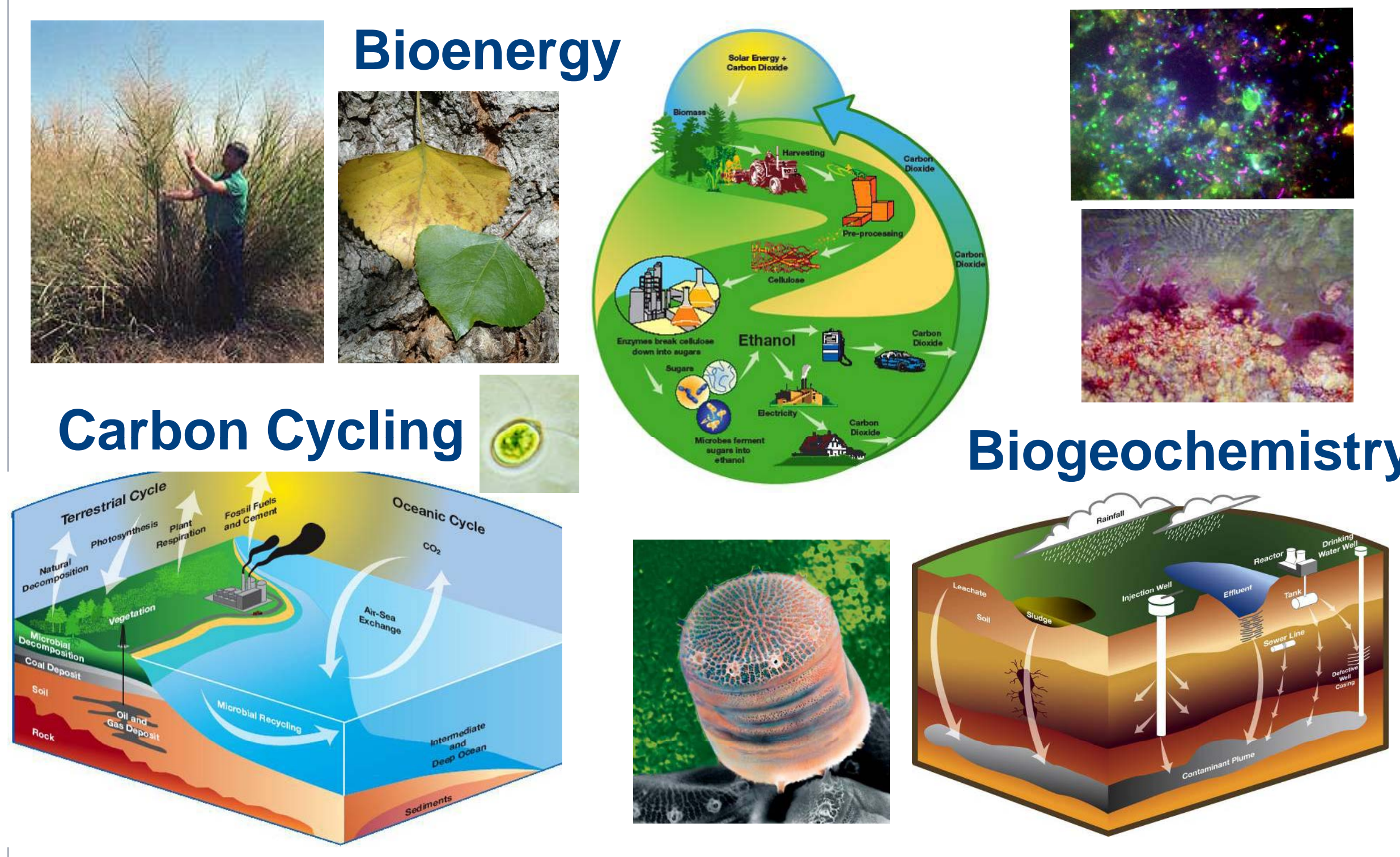


\section{JCIS Oil as Fuel: Big Picture Problem}
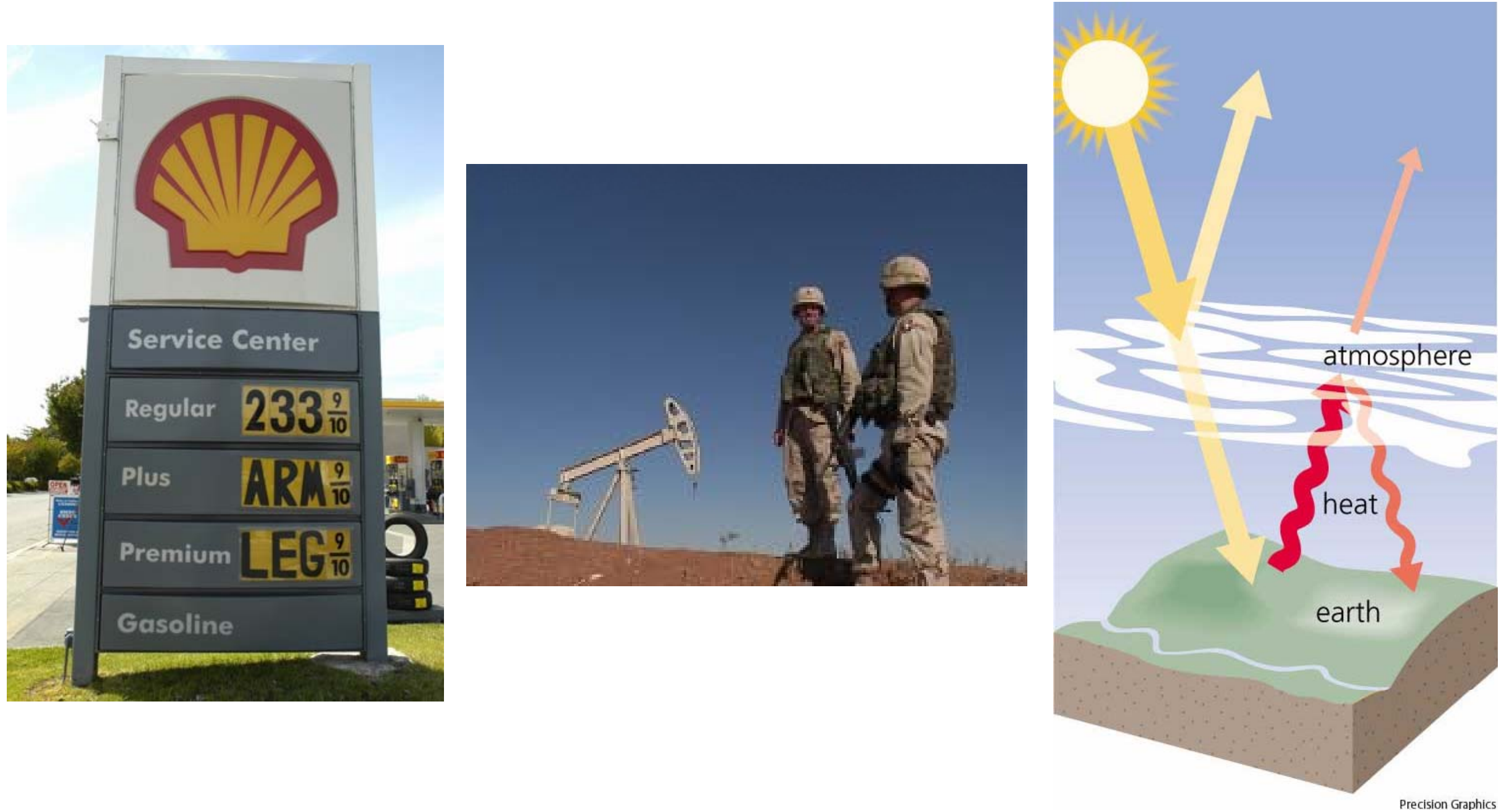


\section{JCI Global Warming: Carbon Cycling}

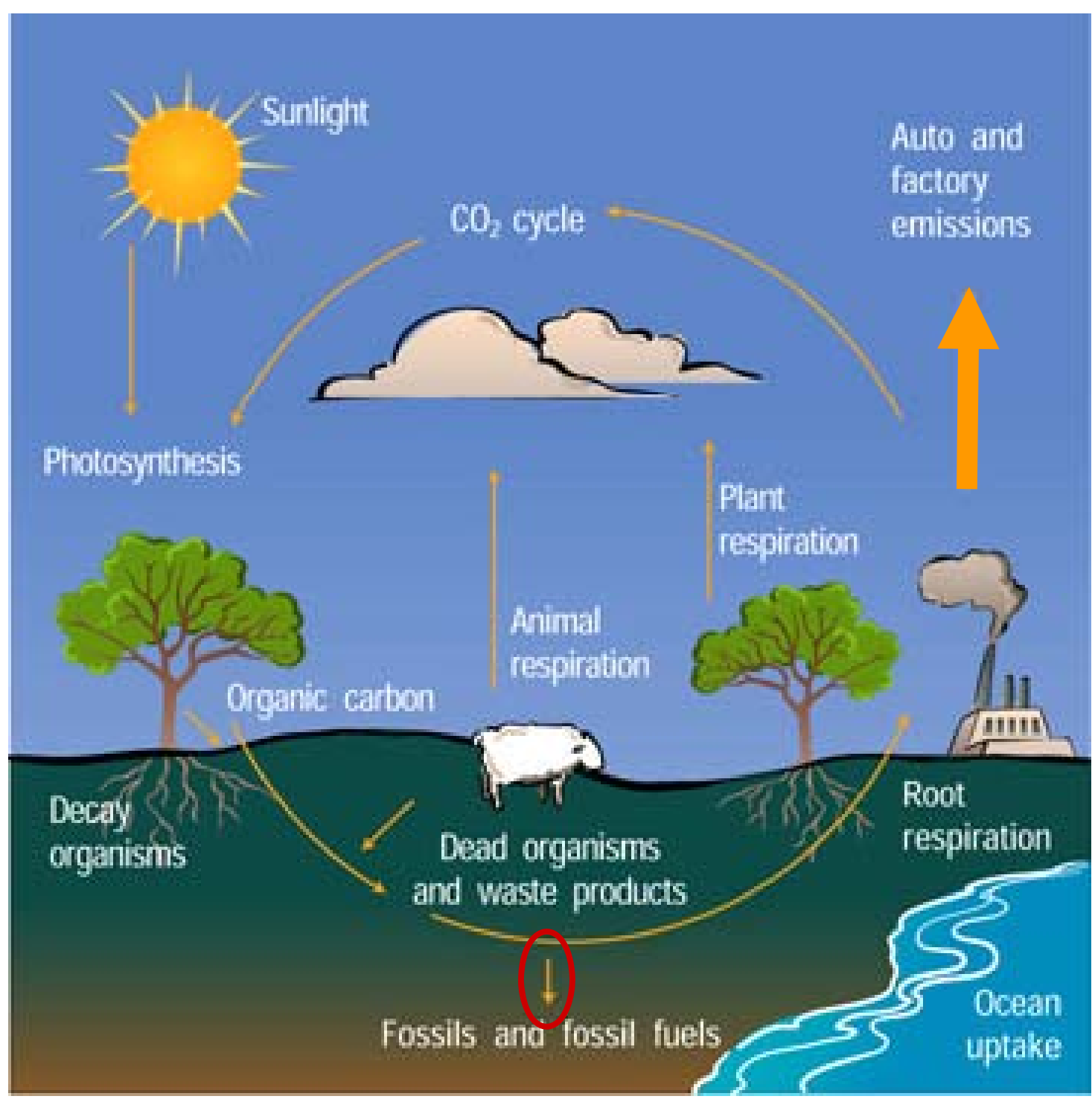



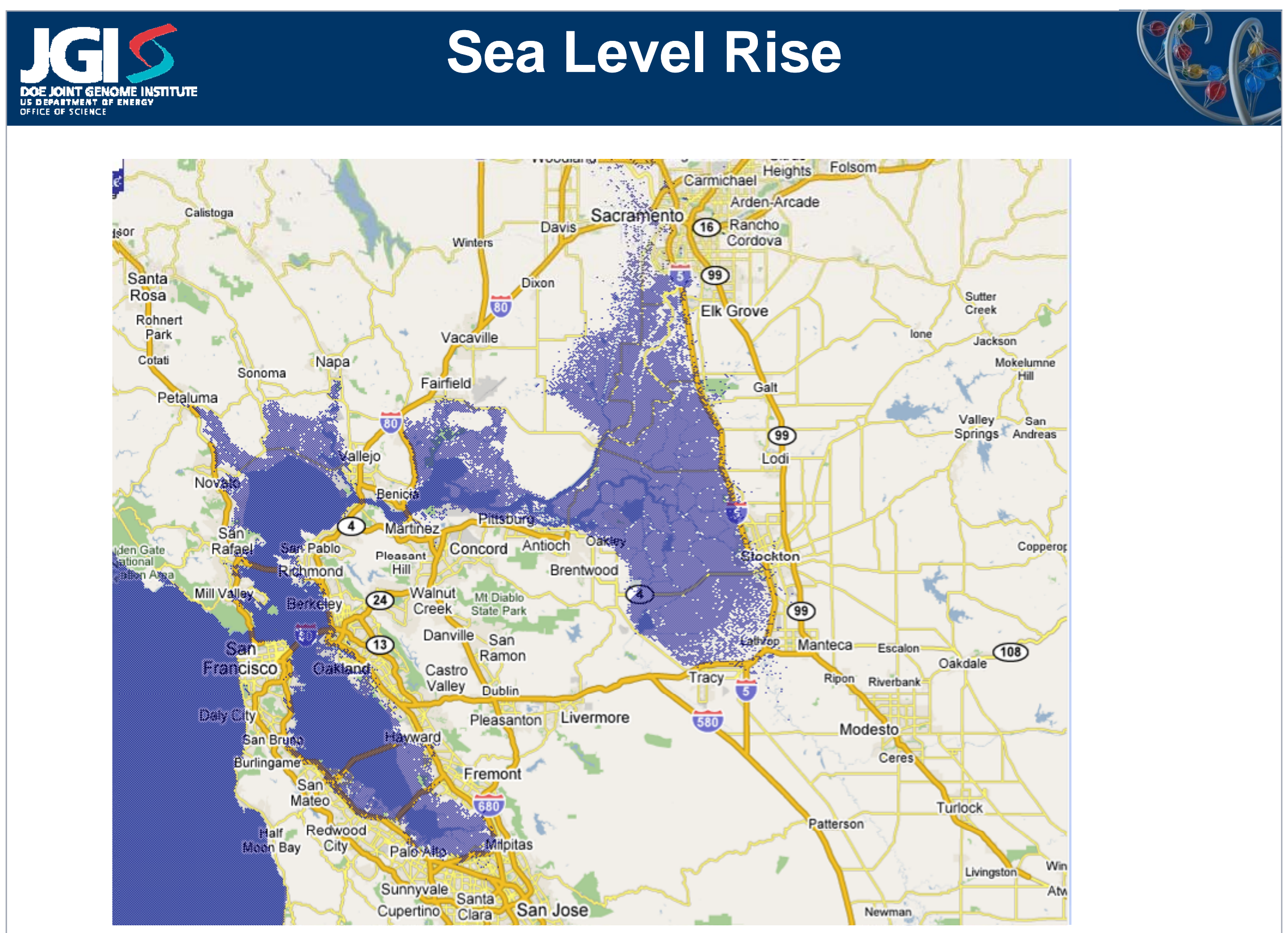


\section{J 5 Microbes at work}
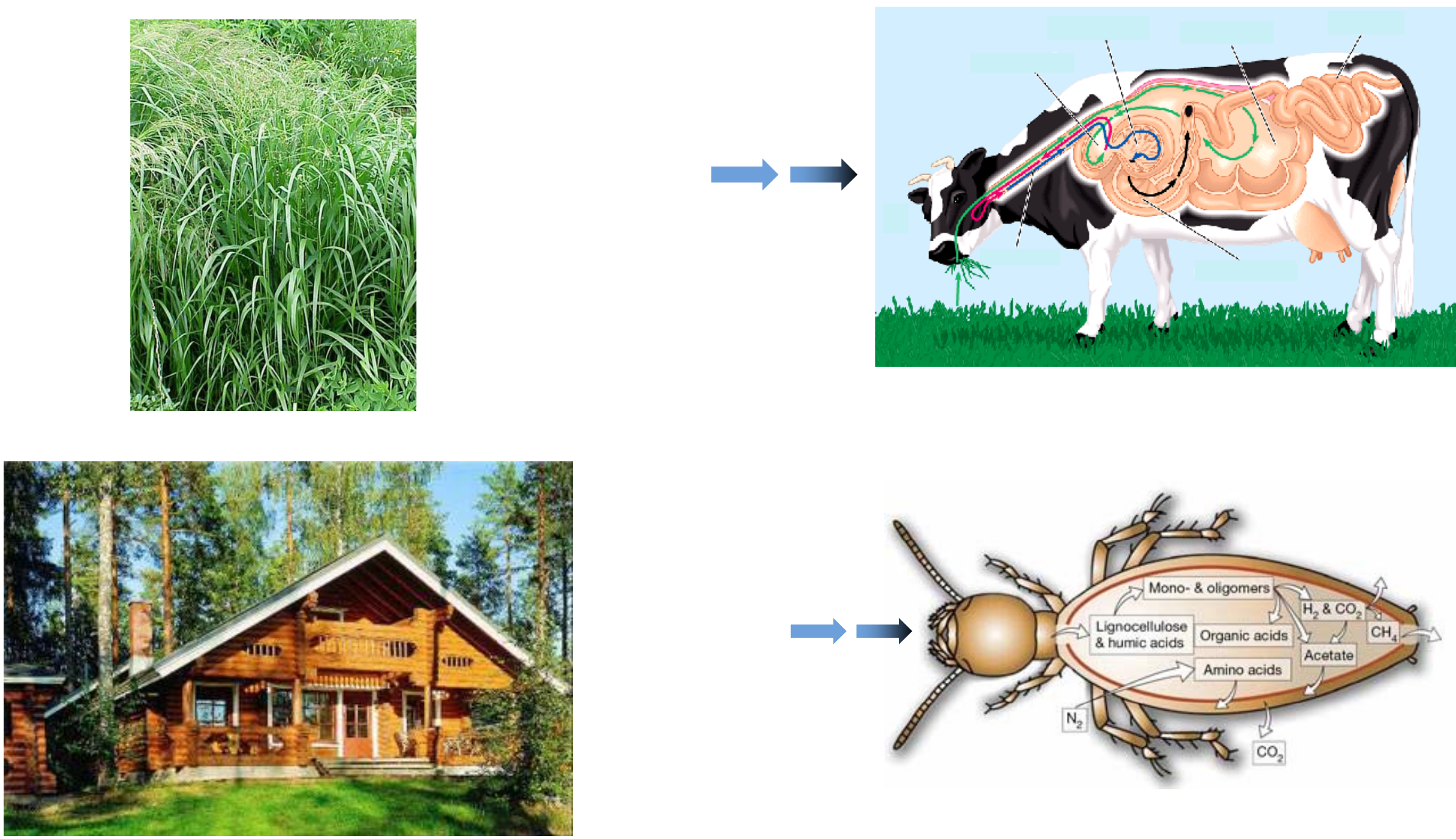


\section{Termites}

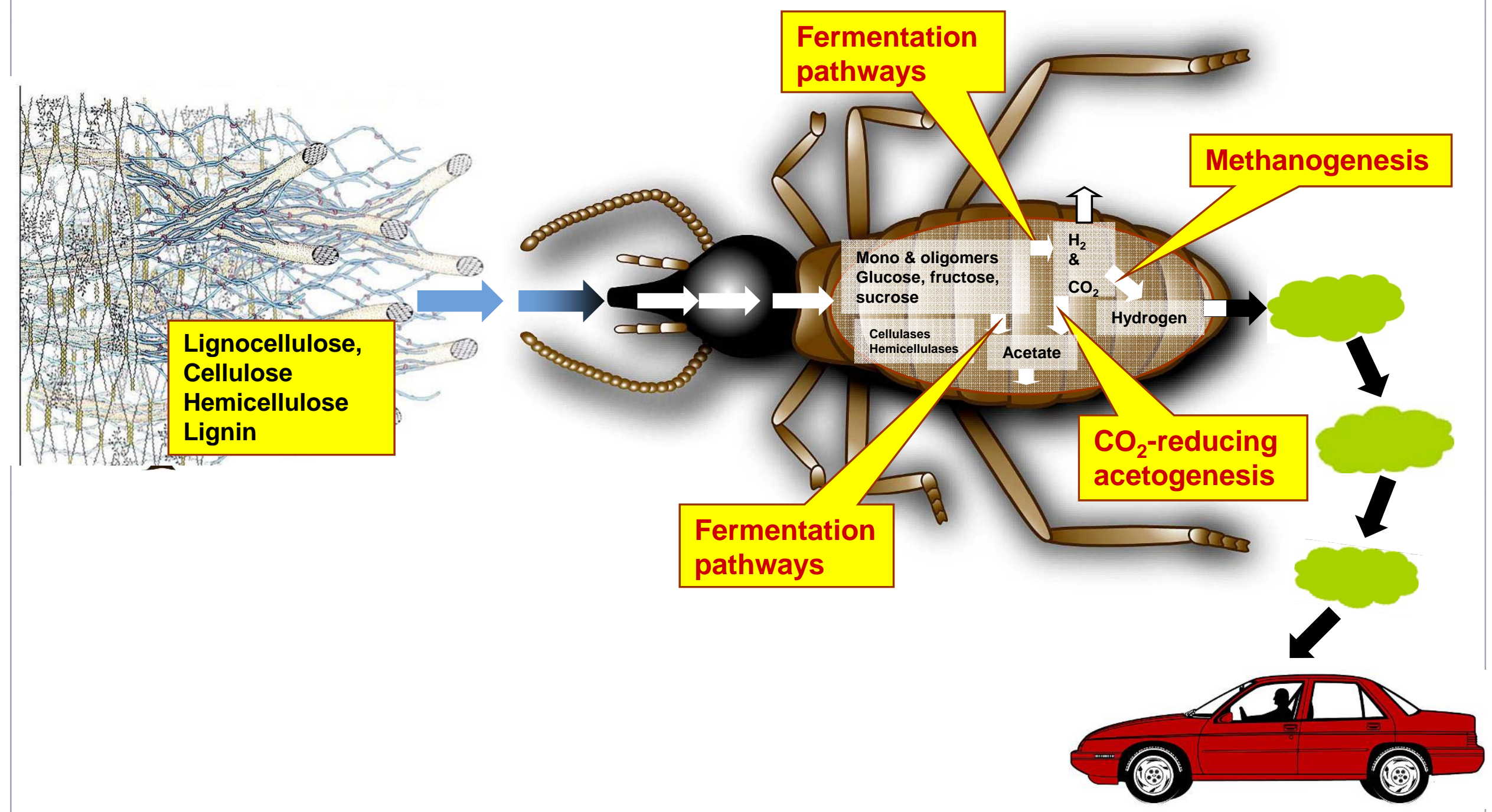

Termites eat wood bacteria in their stomach breaks it down for them which turns into energy 


Studying Microbes
in A Cow's Stomach

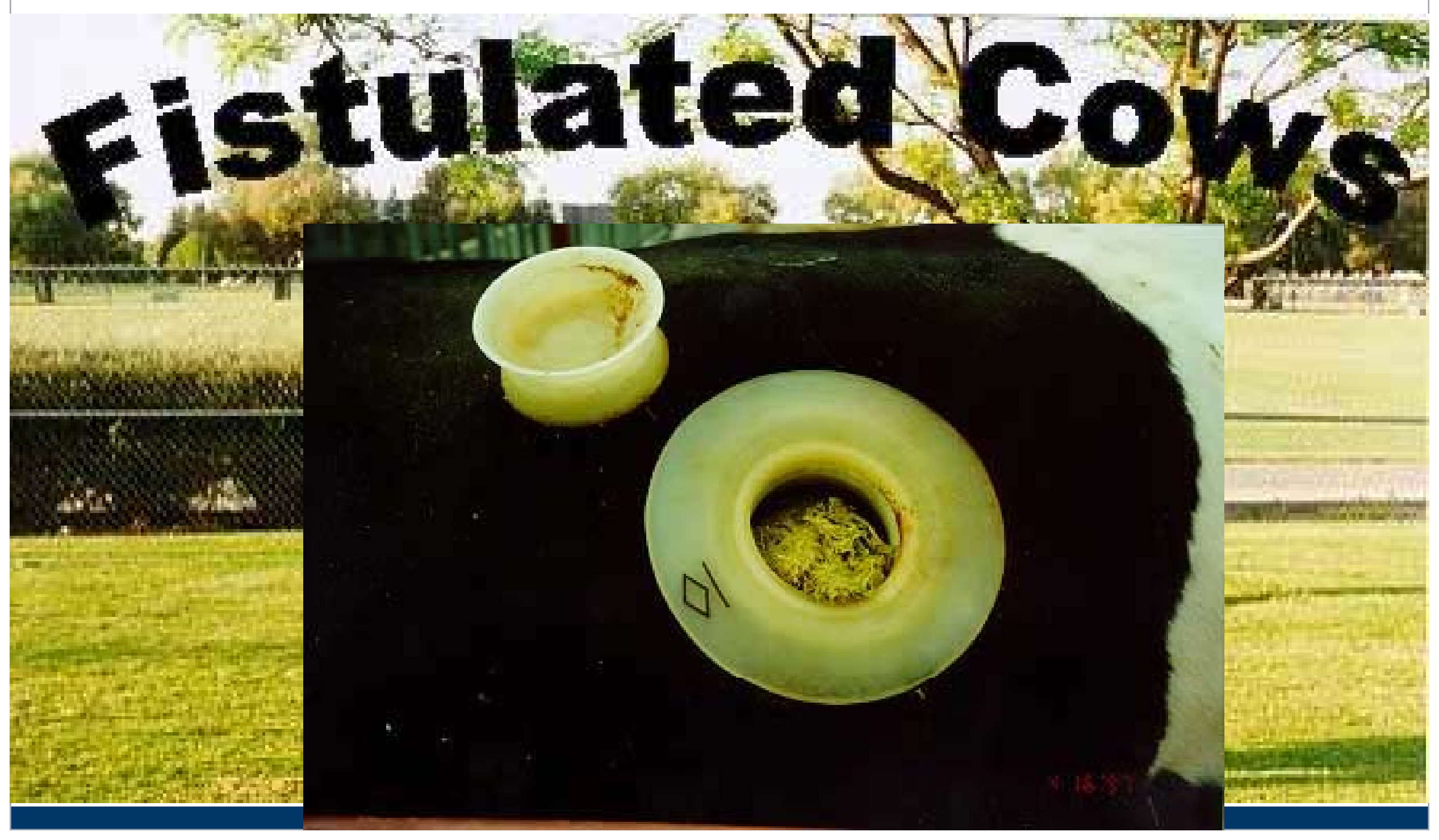




\section{If is a Little Messy \& Smelly!}

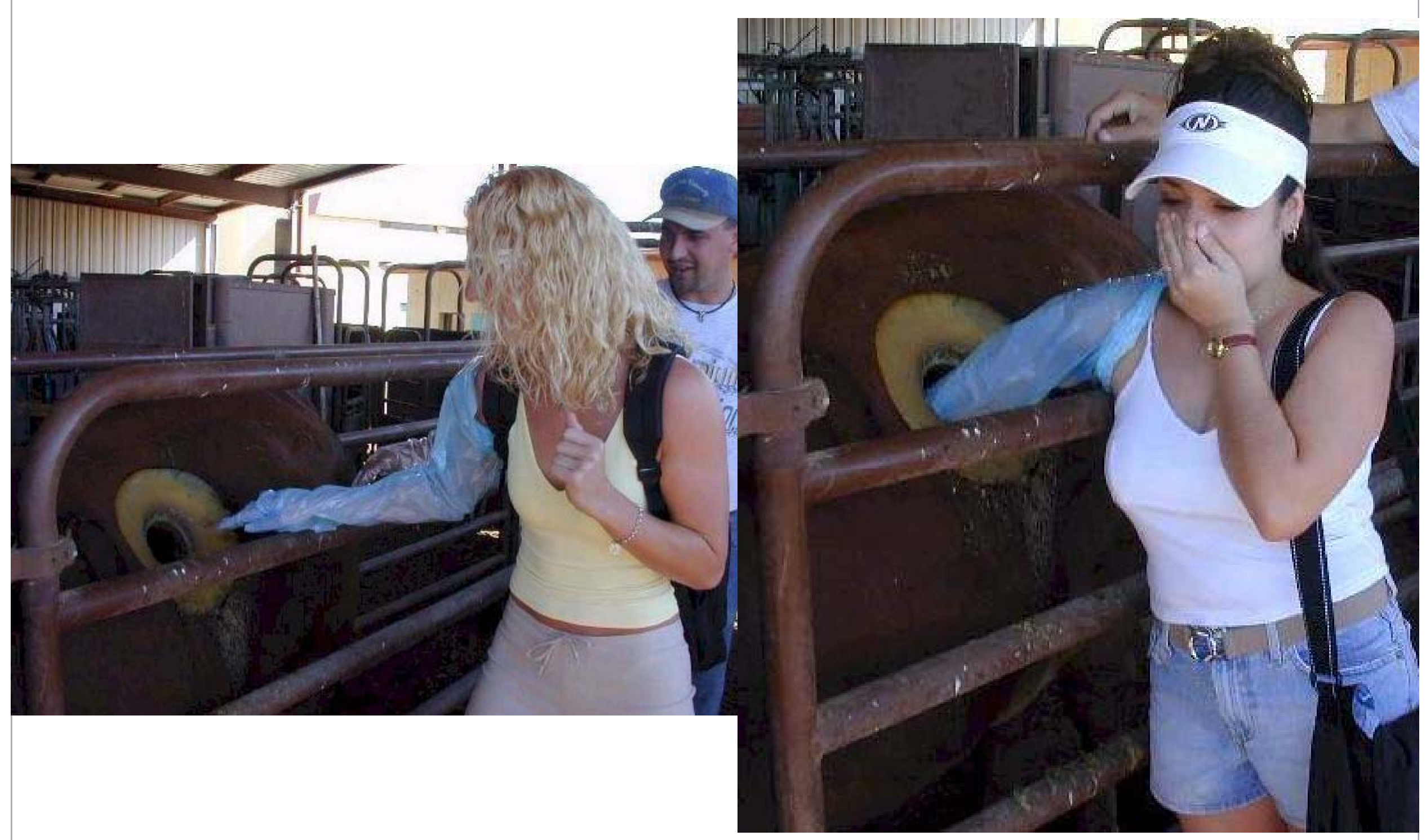




\section{CIS Converting Cellulose to Alcohol}

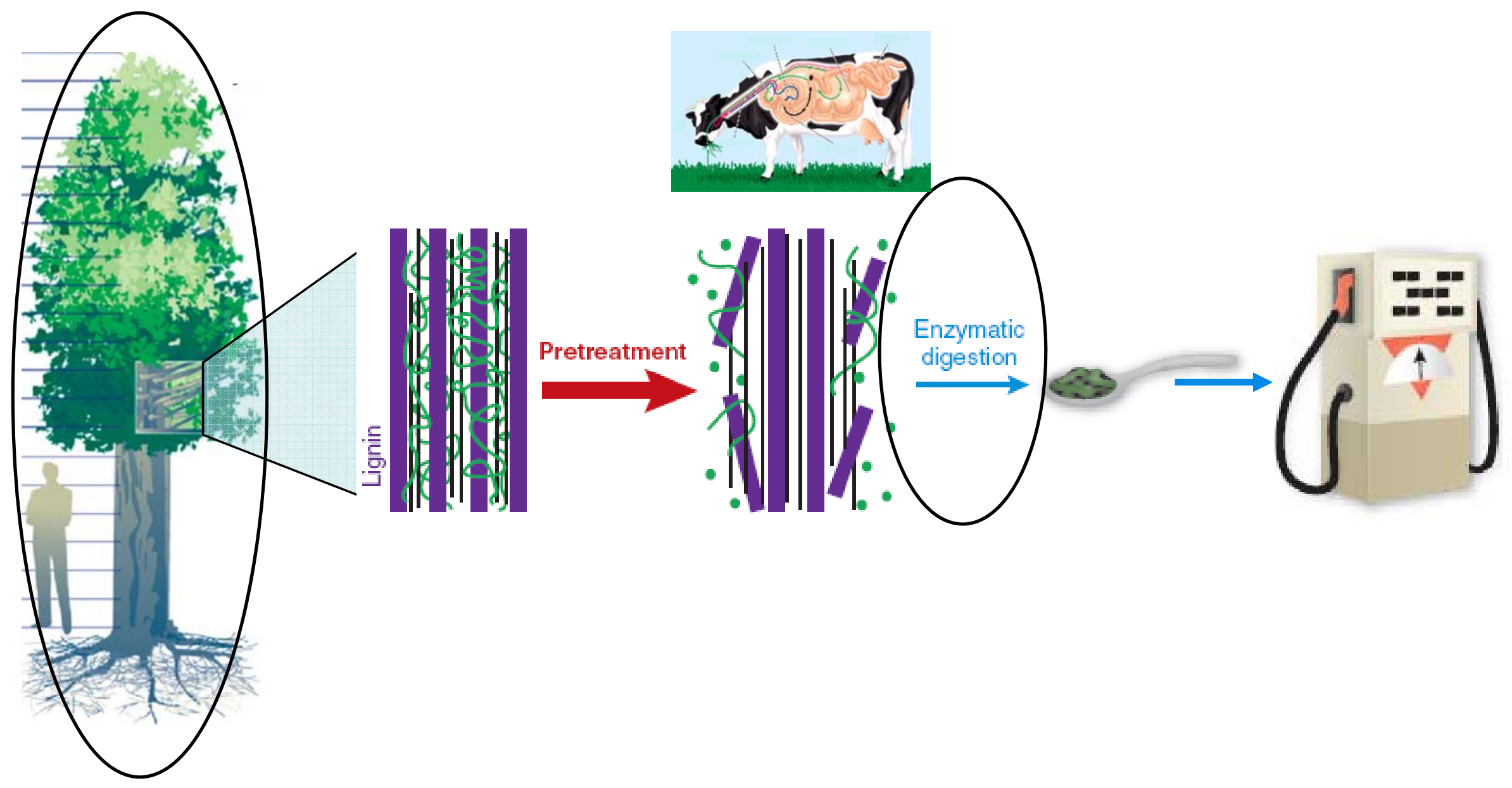



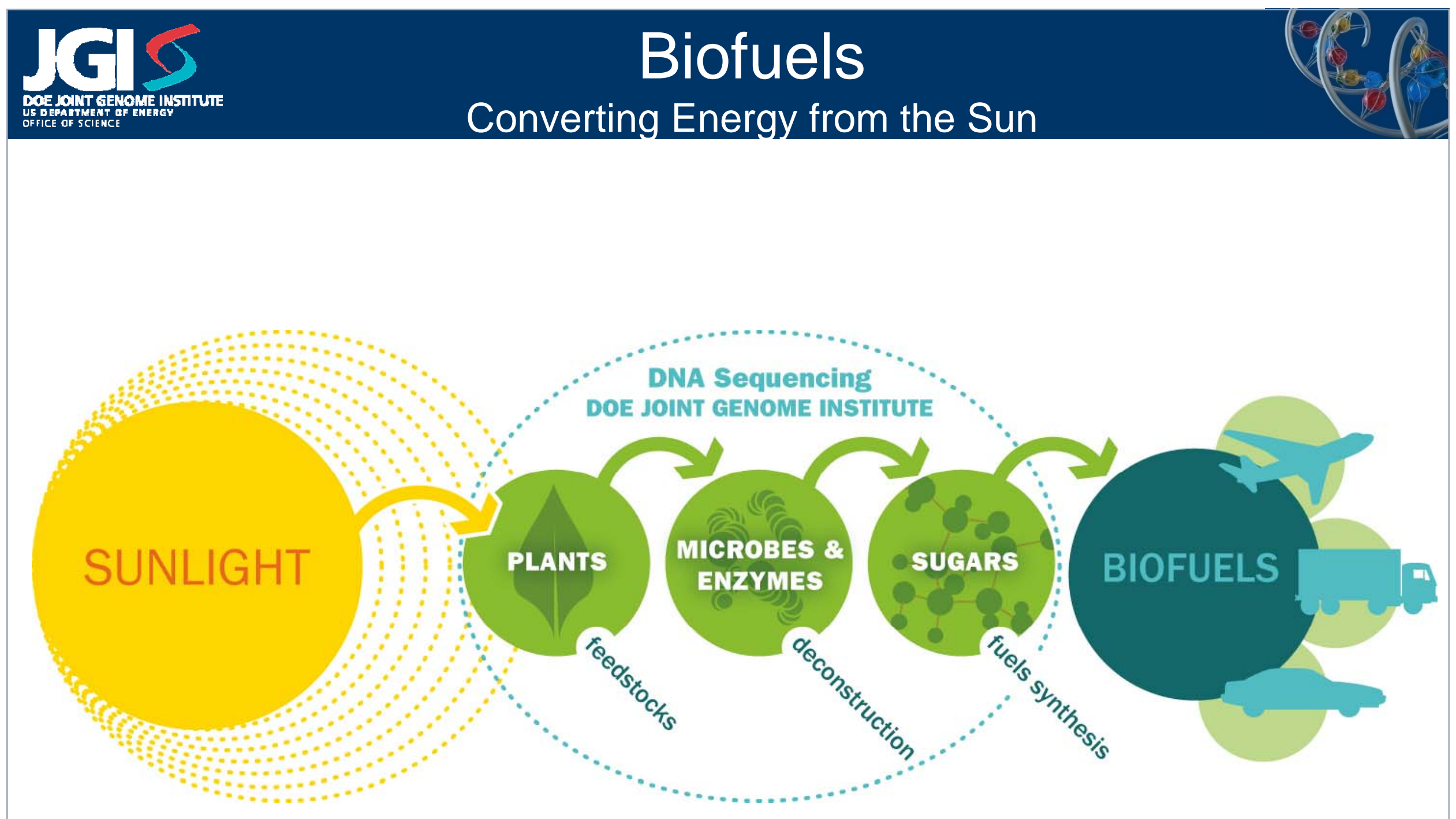

Genomic Strategies for Averting an Energy Crisis and Reducing Global Warming 


\section{How We Work}

Office \& Manufacturing Work Environments

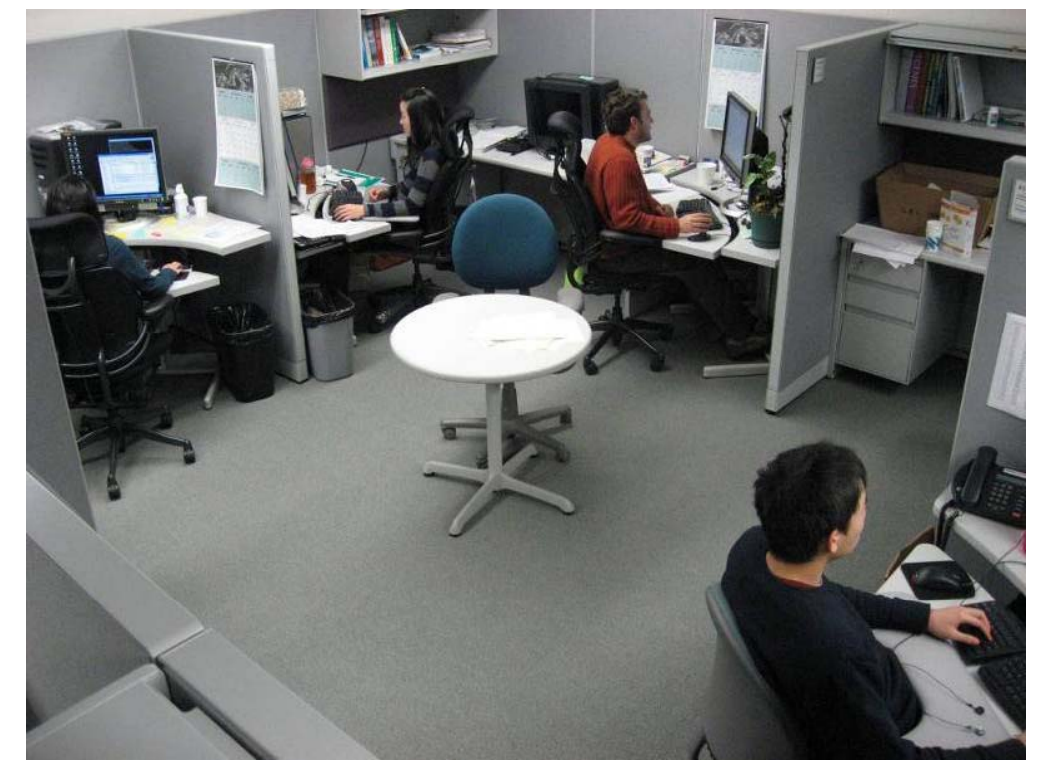

$60 \%$ staff in computerintensive office settings

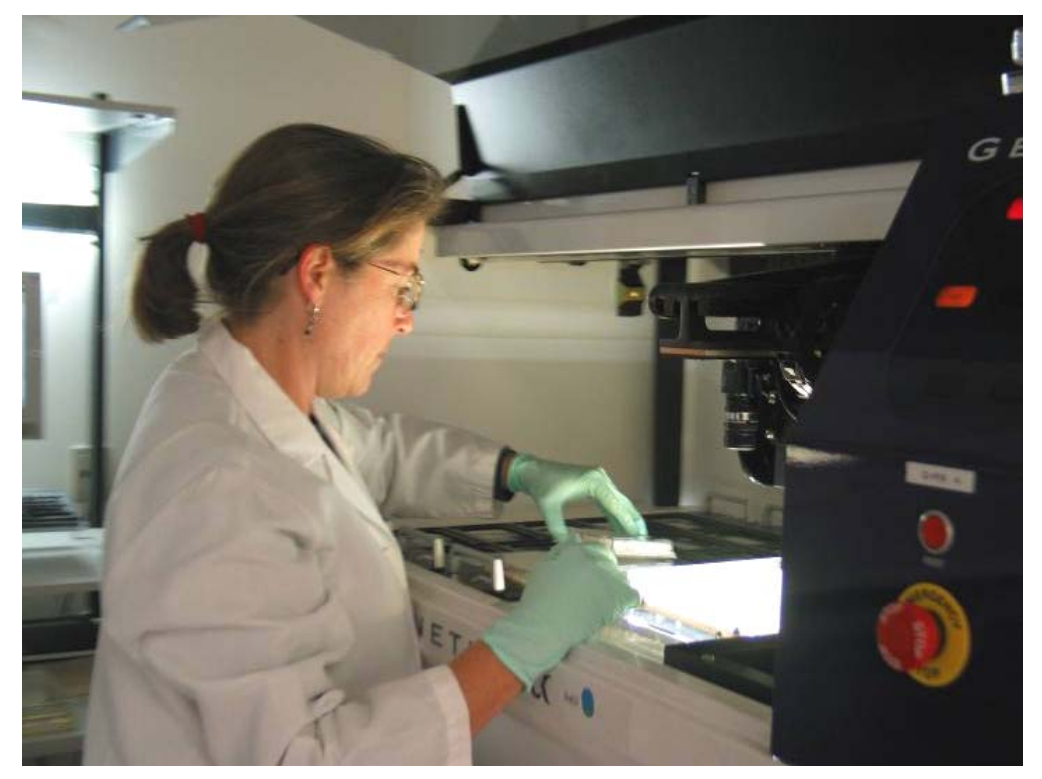

$40 \%$ staff in hand-intensive production tasks (2 shifts) 


\section{JIS \\ Challenges in Biotechnology}

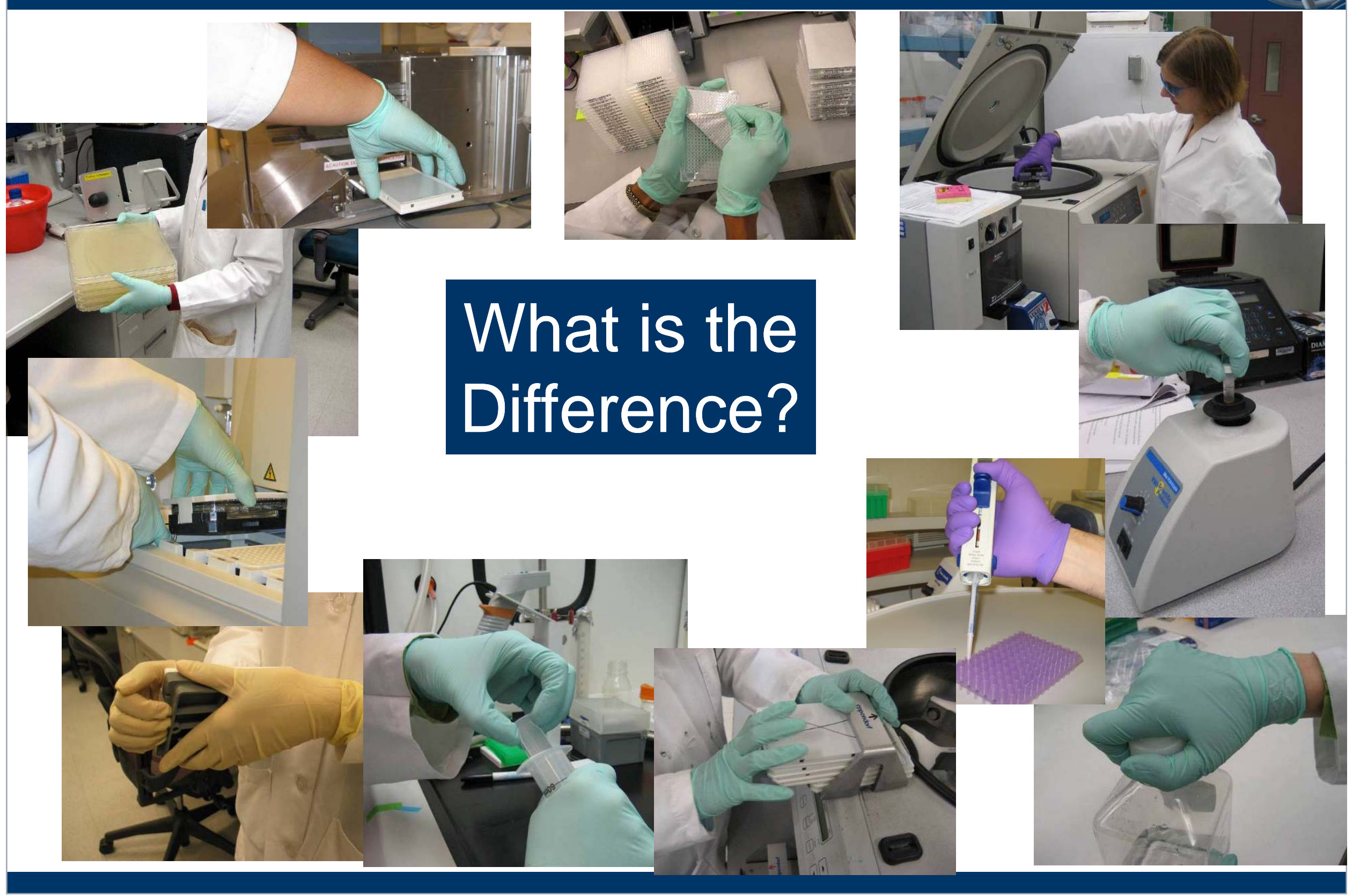




\section{JGI Ergonomics Program}

\section{Prior to Stand down}

- Ergo Working Group (EWG)

- 139+ Ergo projects

- Ergonomists onsite 2x a week

- Early Intervention Program

- Pilot John Muir provider as a satellite health services for LBL employees

- October National Ergonomics Awareness Month 


\section{Ergo Project Status}

Prior to Stand down

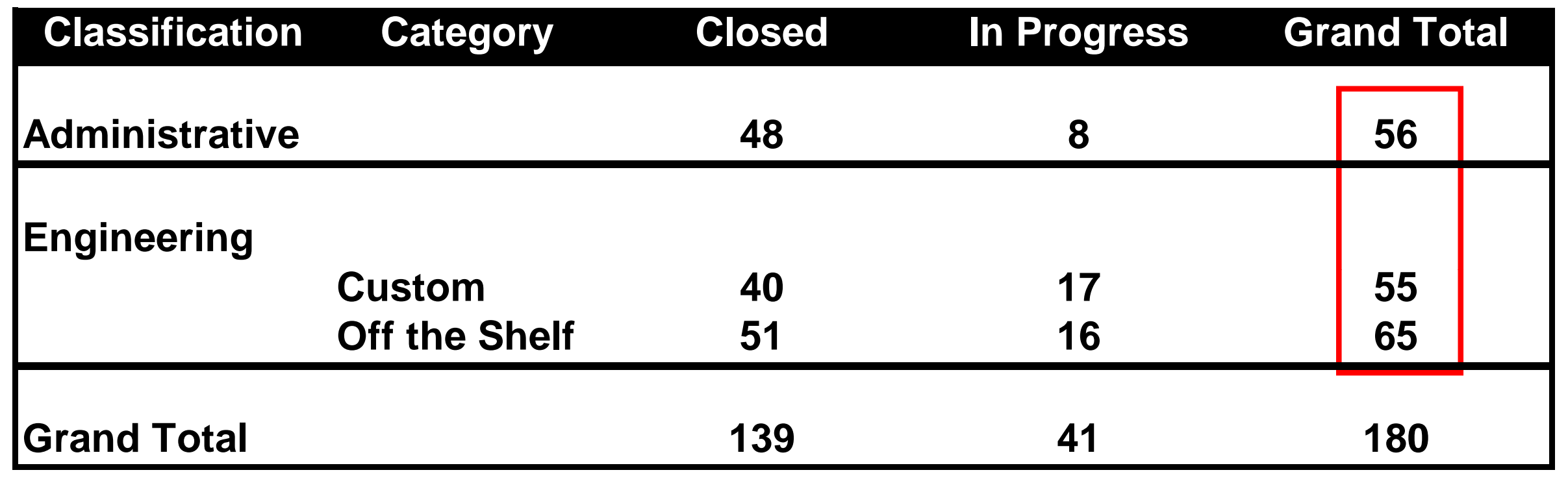

\# Ergo Projects by Classification 


\section{(C) \\ Top 3 High Risk Factor Tasks}

Thermal Cycler Loading
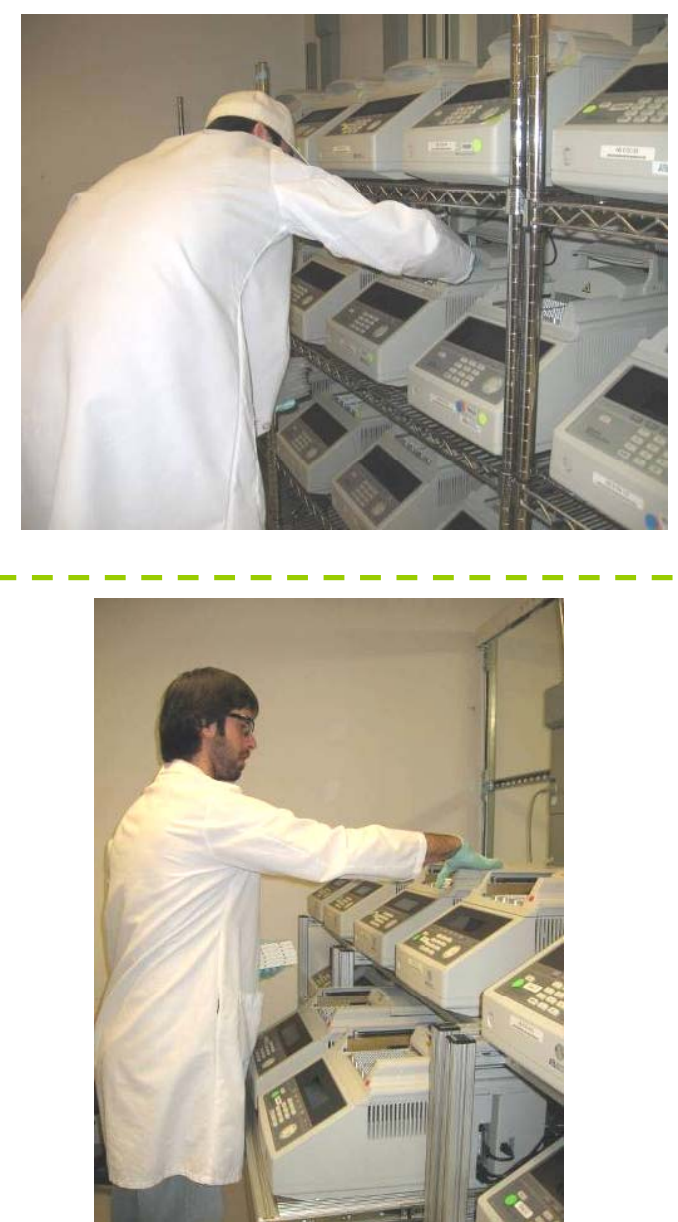

Peeling Seals
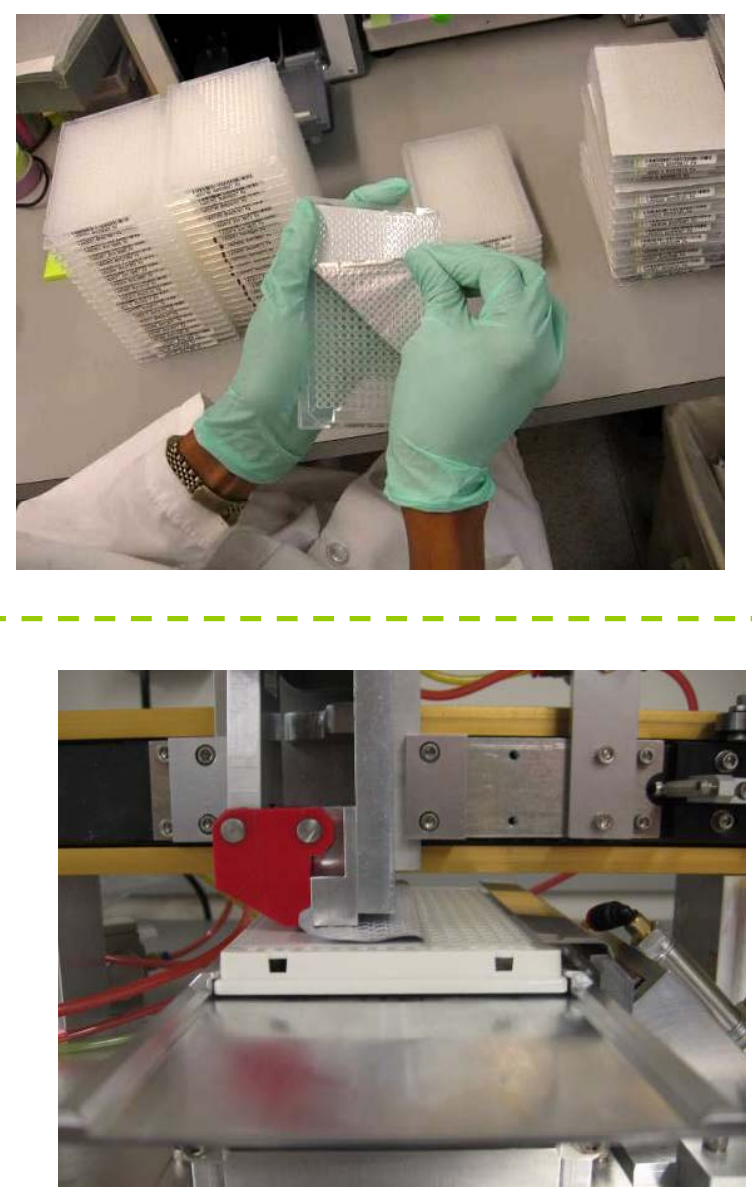

Freezer Rack Lifting
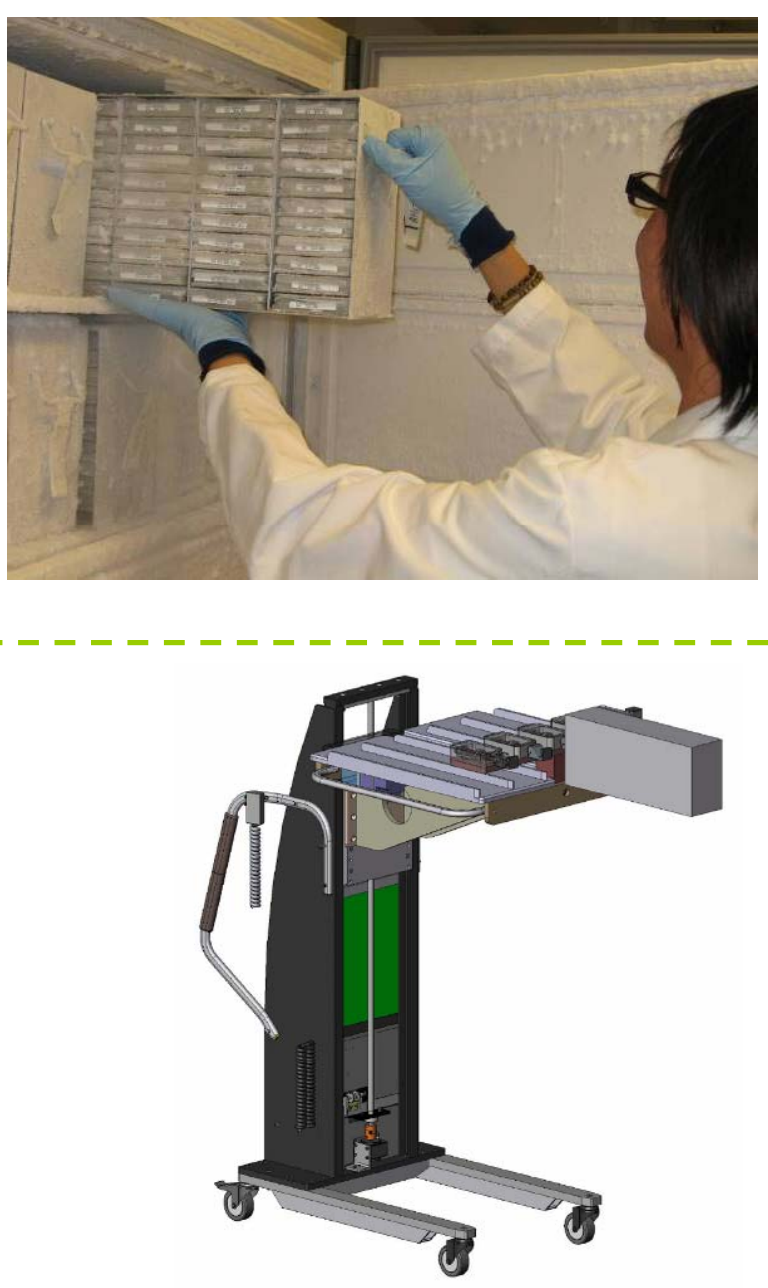


\section{CISProduction Wide Ergo Improvements

Height-Adjustable \& Custom Designed Tables
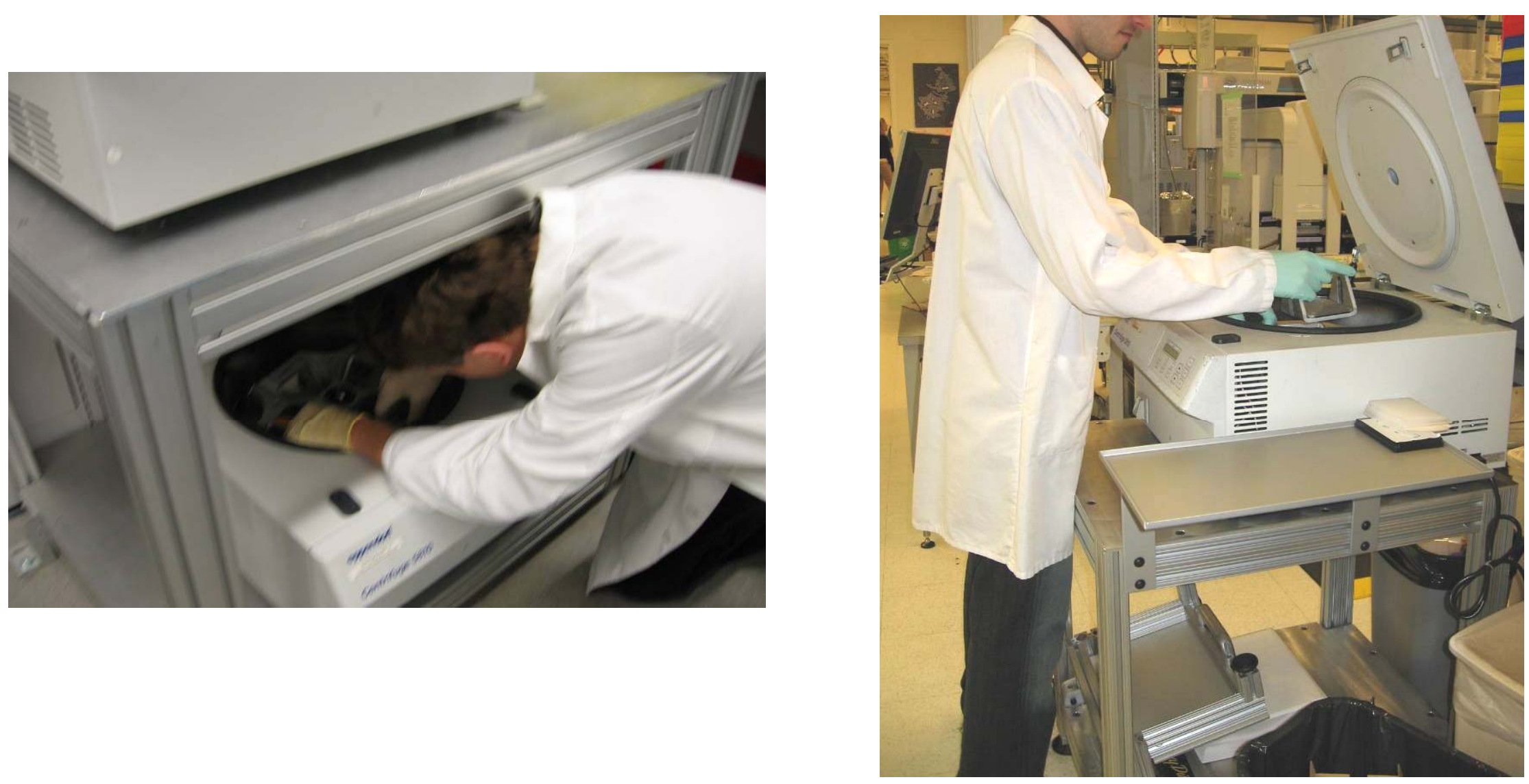

Before

After 


\section{LISProduction Wide Ergo Improvements

\section{Best Practices and Training}
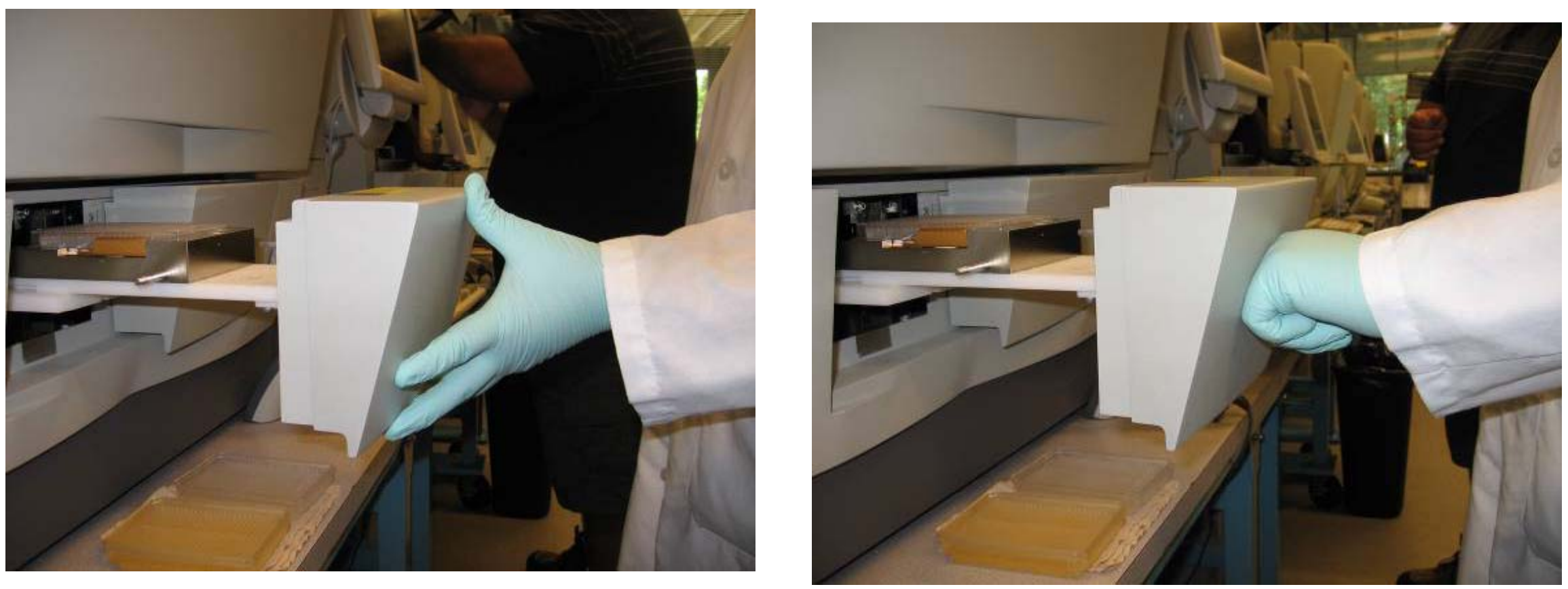


\section{(C) 5 Production Wide Ergo Improvements}

Anti-Fatigue Mats

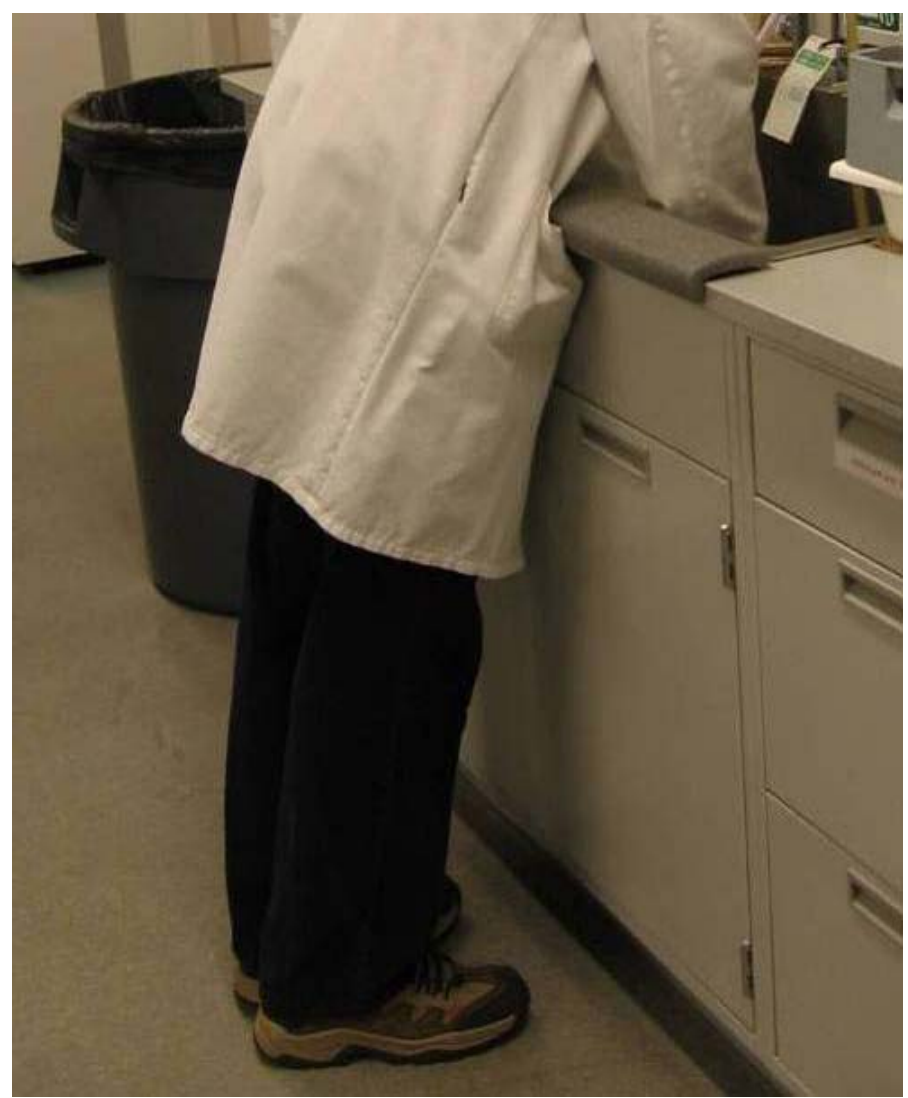

Before

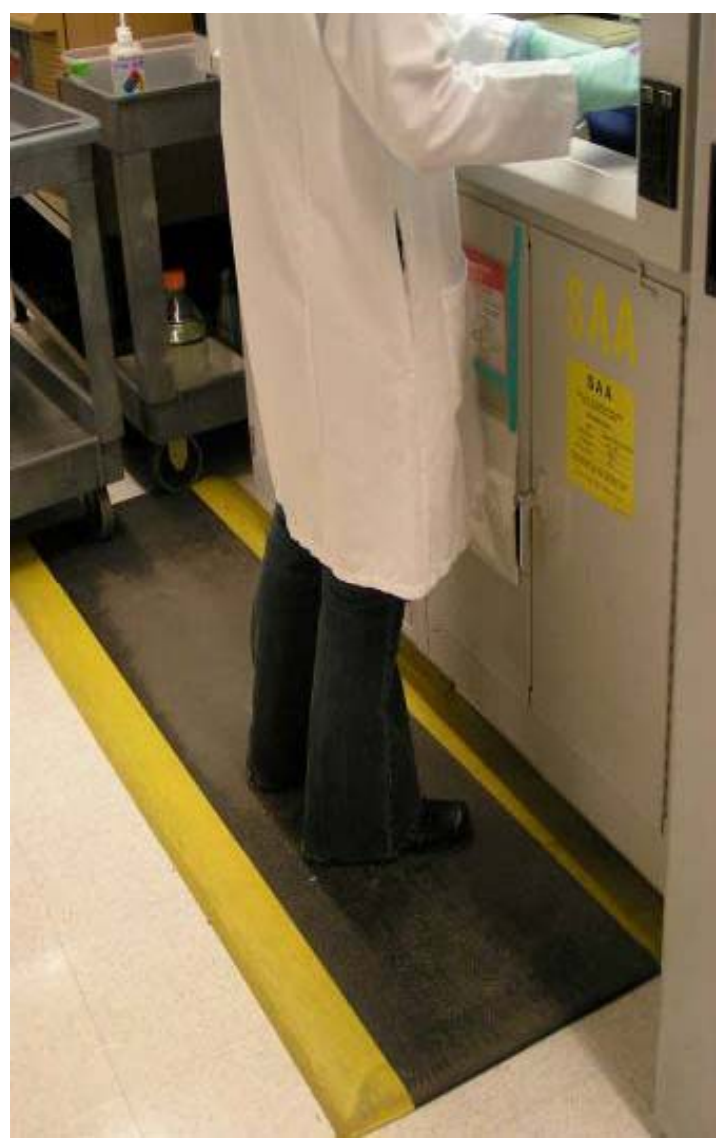

After 


\section{JCIS \\ 2007 Ergo Cup Winners "Team Driven Workplace Solutions"}

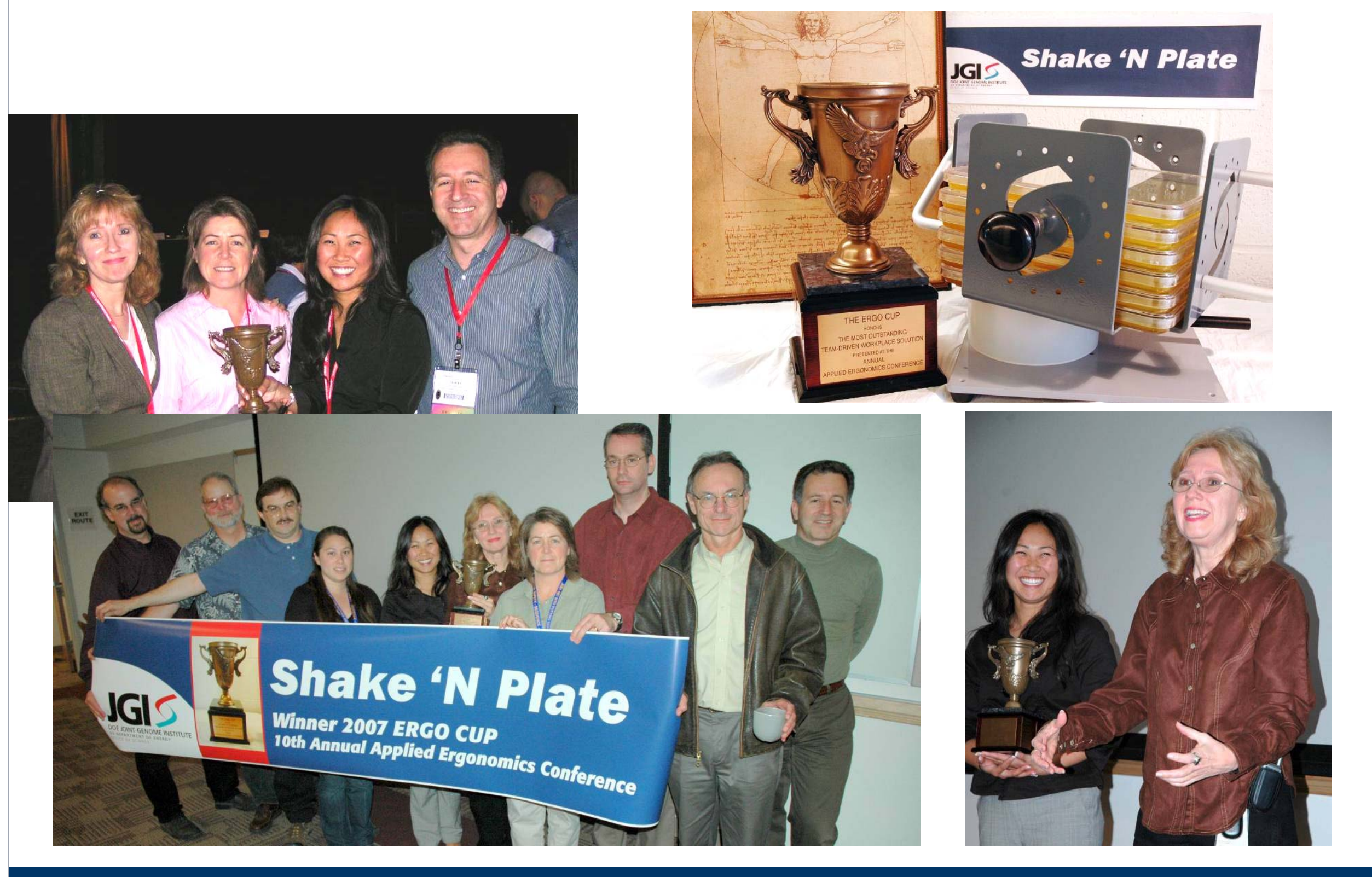




\section{Recordable Injury History}

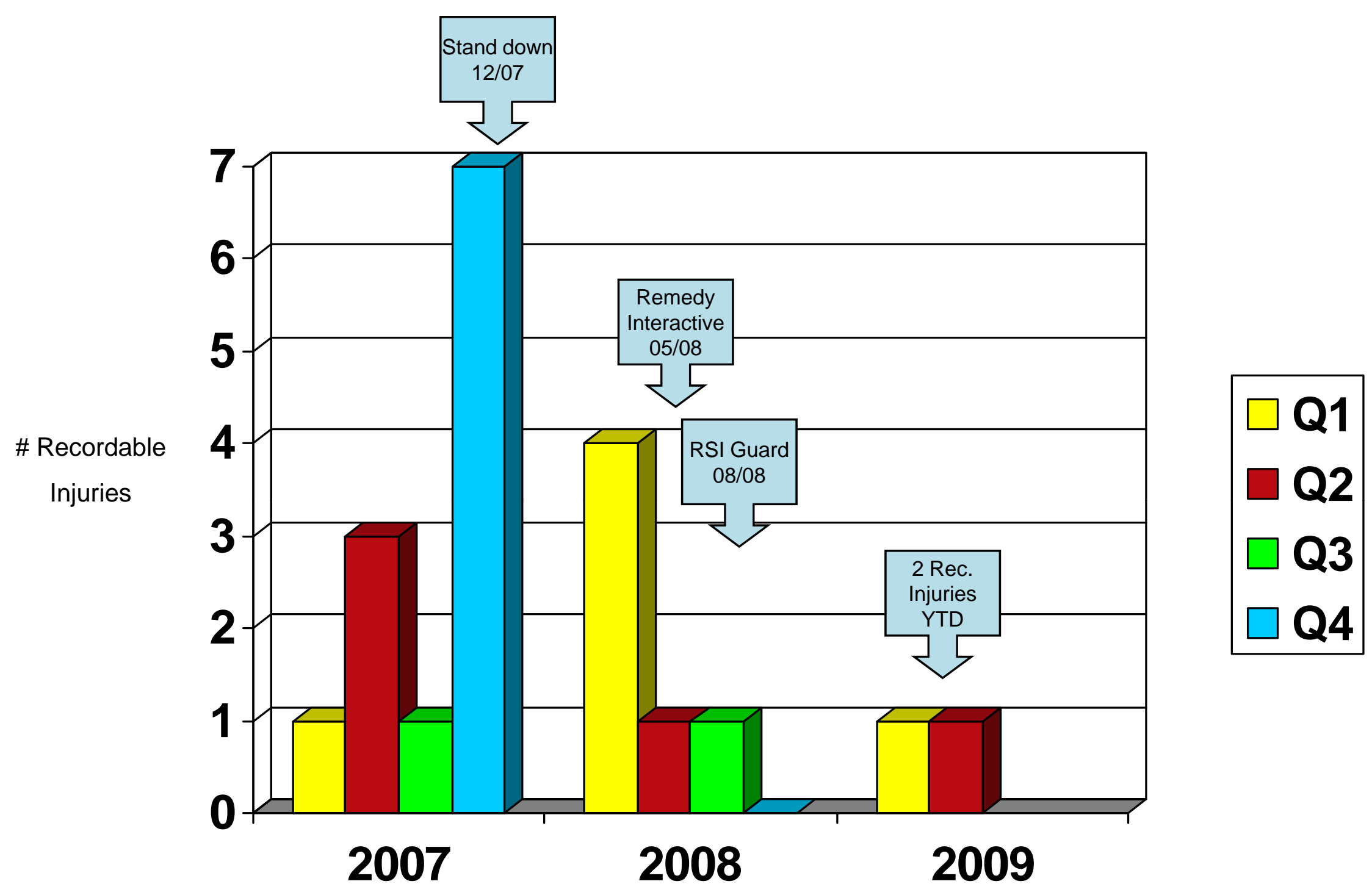


1) Job hazard analysis and evaluation of work process in RCA are less than adequate

2) Formal management walk around program at the JGI is not adequately identifying or controlling ergonomic hazards

3) Exposure of workers to ergonomic hazards in the RCA is excessive

4) JGI processes for worker involvement in safety needs improvement 


\section{JGI Observations}

- Too many people in early intervention

- Communication with medical at partner labs needed improvement

- Partner lab Contract Transition (stress)

- Communication with employees was difficult (trust)

- Rotating staff for cross-training

- Morale

- Repetitive stress

- Aspects of the Production Org structure were not working

- Next generation sequencing technologies were being introduced (very manual processes/stress knowing one line will be shut down?) 


\section{Ergonomics Stand Down}




\section{Stand Down/Stand Up}

Goal: To provide an injury-free workplace, so that at the end of the day employees could go home without experiencing any discomfort.

How:

- Understanding how our current morale problems are negatively impacting safety

- Improve communication and teamwork to create a safer work environment

- Involve EVERYONE in seeking the solutions

- Identify all hazards within the production process

- Implement necessary changes to ensure a safe work environment. 


\section{"December Stand Down"}

\section{Who was involved?}

- Onsite consultant

- Macroergonomics \& Human and Organizational Change

- 2 Ergonomists/Therapists

- IE (industrial engineer)

- JGI Safety Officer and EHS Division Safety Coordinator

- JGI Director, Operations, HR 


\section{Example of Daily Schedule}

Week 3 Schedule

\begin{tabular}{|c|c|c|c|c|}
\hline 17 & 18 & 19 & 20 & 21 \\
\hline \multicolumn{5}{|c|}{ Kick-off } \\
\hline $\begin{array}{l}\text { Working } \\
\text { Groups II }\end{array}$ & $\begin{array}{l}\text { Working } \\
\text { Groups II }\end{array}$ & $\begin{array}{l}\text { Working } \\
\text { Groups II }\end{array}$ & Working Groups II & Debrief \\
\hline Lunch & Lunch & Lunch & Lunch & Eddy Lunch \\
\hline $\begin{array}{l}\text { Working } \\
\text { Groups II }\end{array}$ & $\begin{array}{l}\text { Working } \\
\text { Groups II }\end{array}$ & $\begin{array}{c}\text { Early } \\
\text { Intervention } \\
\text { Brainstorming }\end{array}$ & $\begin{array}{c}\text { Safety } \\
\text { Checklist/Walkabo } \\
\text { ut } \\
\text { Training }\end{array}$ & Debrief \\
\hline $\begin{array}{c}\text { Daily } \\
\text { Summary }\end{array}$ & $\begin{array}{c}\text { Daily } \\
\text { Summary }\end{array}$ & $\begin{array}{c}\text { Daily } \\
\text { Summary }\end{array}$ & $\begin{array}{c}\text { Debrief } \\
\text { (Ira/Andy) }\end{array}$ & $\begin{array}{l}\text { Actions for } \\
\text { Shutdown } \\
\text { Return }\end{array}$ \\
\hline Nurse Meeting & Nina Rosenberg & $\begin{array}{c}\text { Andy Imadd } \\
\text { R. DeBusk }\end{array}$ & $\begin{array}{c}\text { Andy Imada } \\
\text { R. D\&Busk }\end{array}$ & $\begin{array}{l}\text { Andy Imada } \\
\text { Eddy Rubin }\end{array}$ \\
\hline
\end{tabular}

Daily Schedule: $8: 30 \mathrm{am}-5: 00 \mathrm{pm}$

Breaks: 11:00am - 11:15am; 3:00pm - 3:15pm

Lunch: 12:30 - 1:00pm (except for Fri: 12:00 - 1:00pm)

Micro-breaks are to be taken throughout the day 


\section{JCI \\ Daily Assessment of Progress}

I December Safety Stand Down

Week One Summary - End of the Day Assessments

\begin{tabular}{|c|c|}
\hline \multicolumn{2}{|c|}{ 12/04/2007 End of the Day Assessment } \\
\hline+ & Delta \\
\hline Stnall groups participation & Change groups the second time \\
\hline People opened up & Presenters, eliminate the dup lication; agree on items during micro breaks \\
\hline Re-bonding as a group & Rebekah did a lot of work for the snacks \\
\hline People came up solutions; worked together; goal oriented people & Communicate changes \\
\hline Agre ement on both shifts & Micro bre aks every $1-1.5$ hours \\
\hline Snacks & Highlight the passionate ideas \\
\hline Exercise where $A$ changes something about $B$ & Have smaller sessions with working group follow ups \\
\hline Operators and management took re sponsibility for what they weren't doing & Focus on how operators might change \\
\hline Small group facilitators & Fewer groups in this room \\
\hline Facilitator & Micro bre aks coincide with lunch \\
\hline & Stretch break every hour \\
\hline & Felt rushed during break outs. Either more time or more focused \\
\hline & Facilitators Summarize and valiclate flip charts \\
\hline \multicolumn{2}{|c|}{ 12/05/2007 End of the Day Assessment } \\
\hline ( & Delta \\
\hline ID'd re-org, struggle with challenge & Disorganized, pick te ams faster \\
\hline Different people in different groups & Not enough time for breakout sessions \\
\hline Check off similar info & Poor forecasting \\
\hline Glimpse into others roles & Don't qualify availability \\
\hline Managers mixed in & More micro breaks \\
\hline Creativity & Lack of acknowledgement and positive feedback (during group discussion) \\
\hline Enthusiastic & Sidebar conversations \\
\hline Eddy's question session & Not sticking to schedule \\
\hline \multicolumn{2}{|l|}{ Good participation } \\
\hline \multicolumn{2}{|l|}{ New speakers } \\
\hline \multicolumn{2}{|l|}{ Meeting new people } \\
\hline \multicolumn{2}{|l|}{ More discussion, new speakers } \\
\hline \multicolumn{2}{|l|}{ No resistors } \\
\hline \multicolumn{2}{|l|}{ Morale incre ase } \\
\hline \multicolumn{2}{|l|}{ Recognition by Sue } \\
\hline pizza & \\
\hline
\end{tabular}




\section{Macroergonomics}

- Organizational design and change management

- Knowing where we are and accepting where we are going...

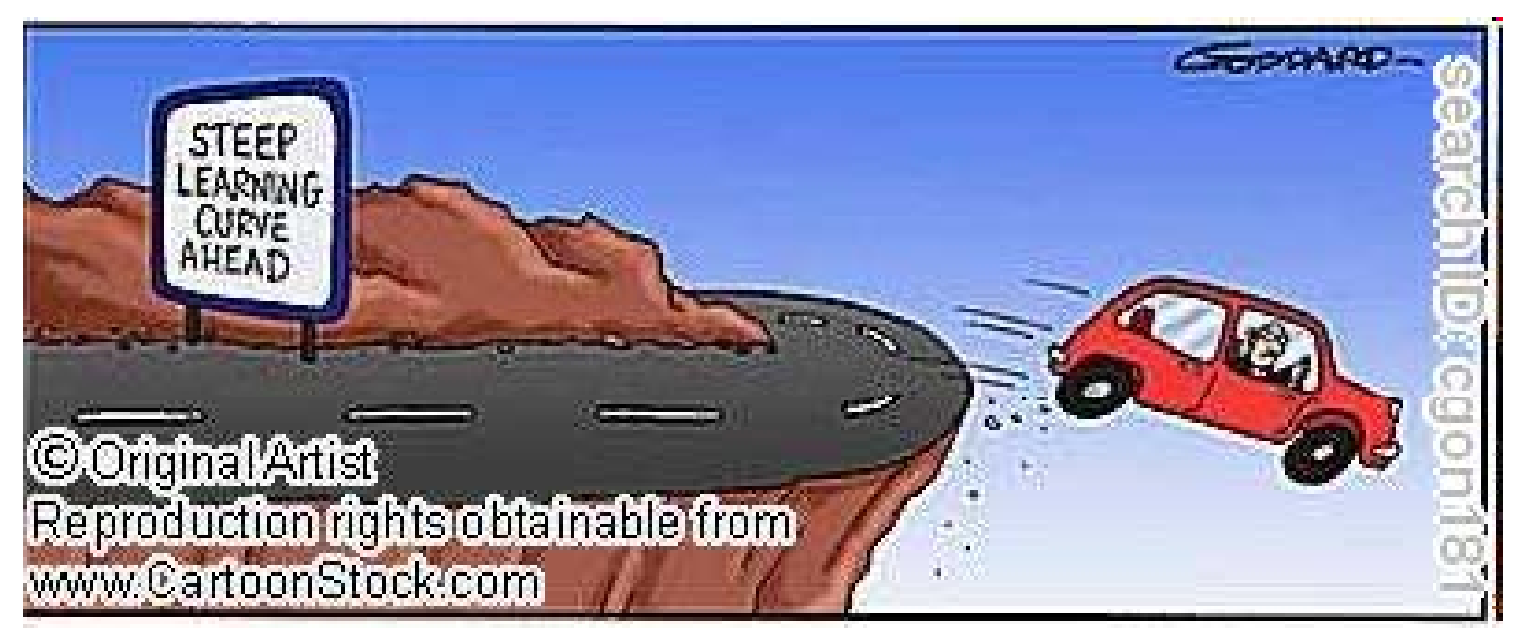




\section{Focus Topics for Case Study Breakout Sessions}

- $\quad$ Ergo Risk Factors

- High Forces: manual process/peeling

- High or Changes in repetition rates: Wellmate to hydra

- Awkward postures

- Training/Familiarity of Process

- Workflow Issues ie. large backlog vs catch up work

- Equipment Malfunctions

- Workload ie. \# batches/person; throughput/shift (how much are you rushing)

- Process Area

- Shift

- Methods of Communication ie. mixed messages 


\section{J Employee Projects During December}

1) Step-by-step Protocol

2) Workflow

3) Risk Assessment

4) Solutions: Administrative, Engineering

5) Required Practices

6) Throughput Model

Use the chemistry area as an example... 


\title{
1) Step by step protocol
}

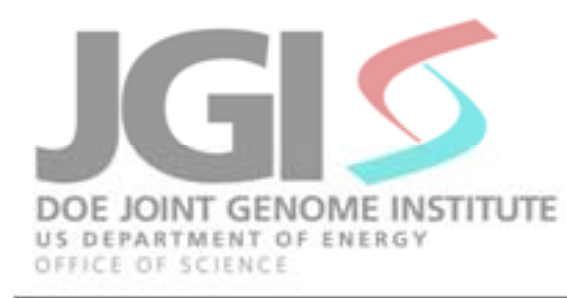

\section{Sequencing Chemistry SOP: Quick Reference Guide}

\author{
Version $\#$ 雍 Date: \\ Author(s): \\ Reviewed Revised by:
}

$12 / 07 / 07$

Gerald Ilog, Catherine Adams, Matthew Zane

Name(s)

\section{Procedure}

$\square$ 1. Check batches for primers and special instructions

2. Pull reagents

Note: Allow one hour to thaw

$\square$ 3. Unseal two batches of RCA plates using automated unsealer.

$\square$ 4. Add 5 ul of Milli $Q$ water to RCA batch 1 Biorad source plates. Take a calibration weight.

Note: Visually check that water is added to each well.

$\square$ 5. Setup Cybio Well Vario. Perfrom a tip QC, tighten tips, perform a tip wash, check dial settings, move tubing, and check lubricant levels.

6. Take a 5 minute Break

7. Unload batch 1 plates from Wellmate ( 16 plates at a time).

8. Load Batch 2 plates on Wellmate.

$\sqcap$ 9. Svin down Batch 1 Biorad vlates. 
 \\ Chemistry Overview Flowchart, Risk Assessment, Engineering solutions}
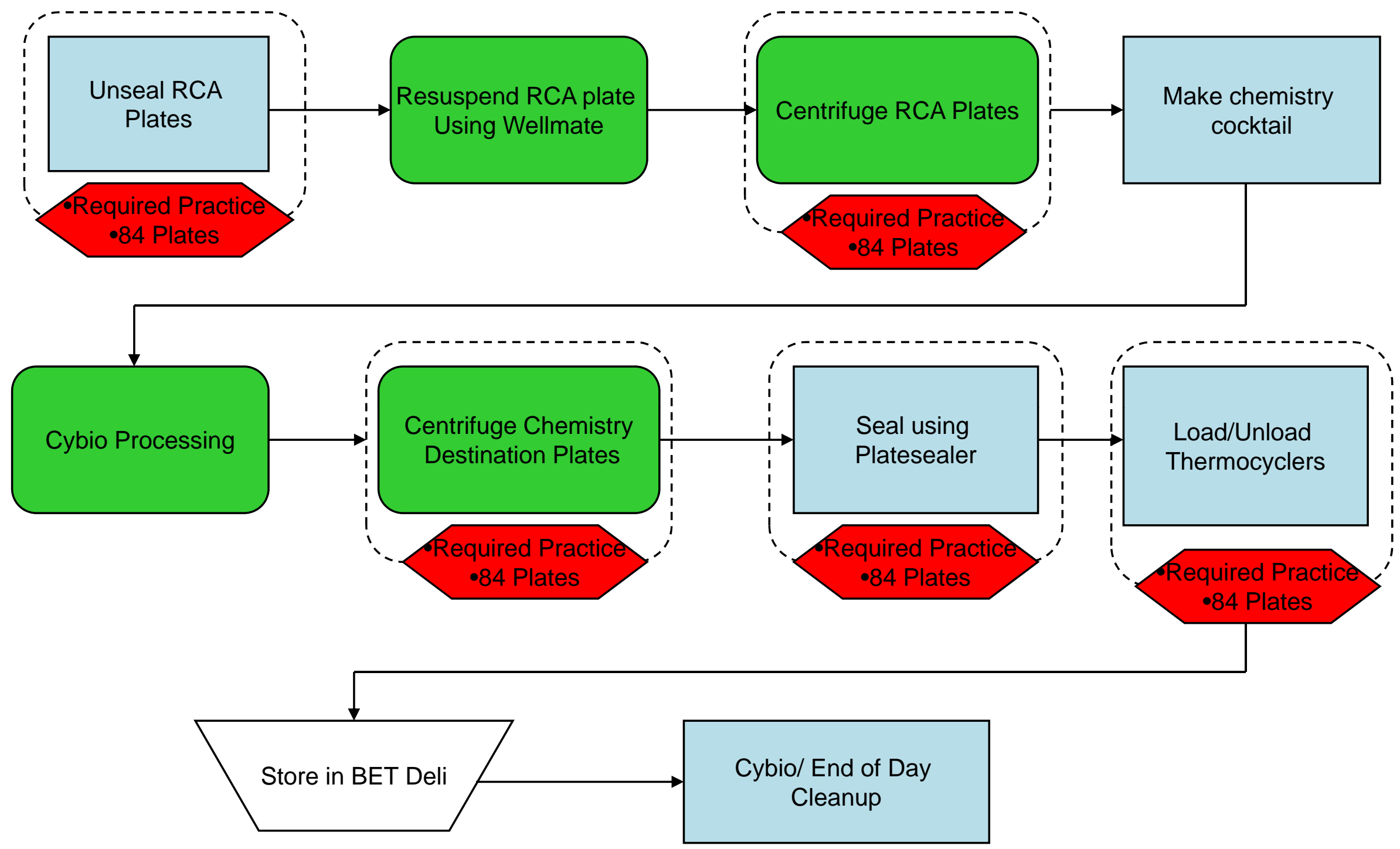


\section{How to use the "It's A Peeling" Button}

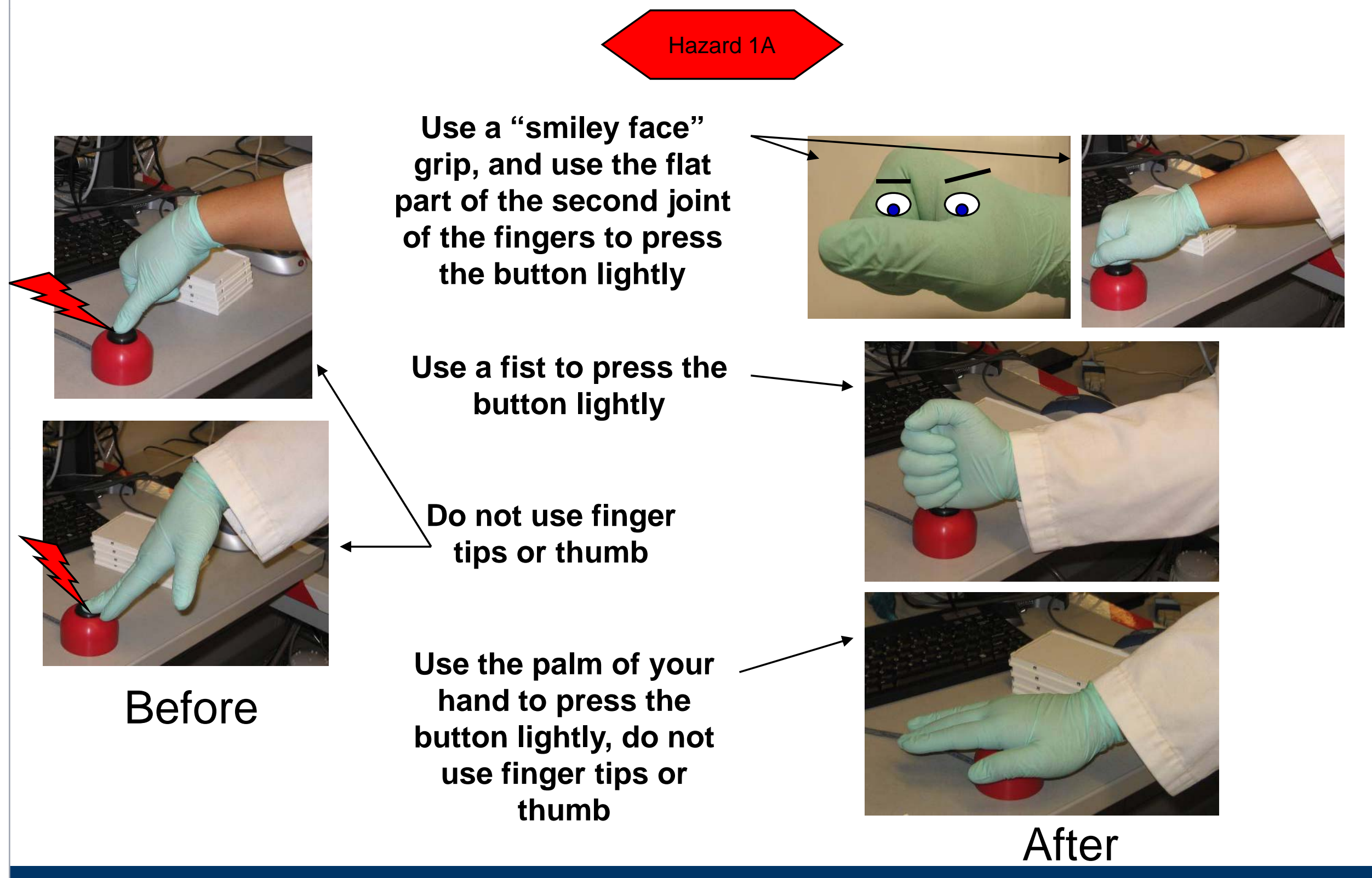




\section{Sealing Plates}
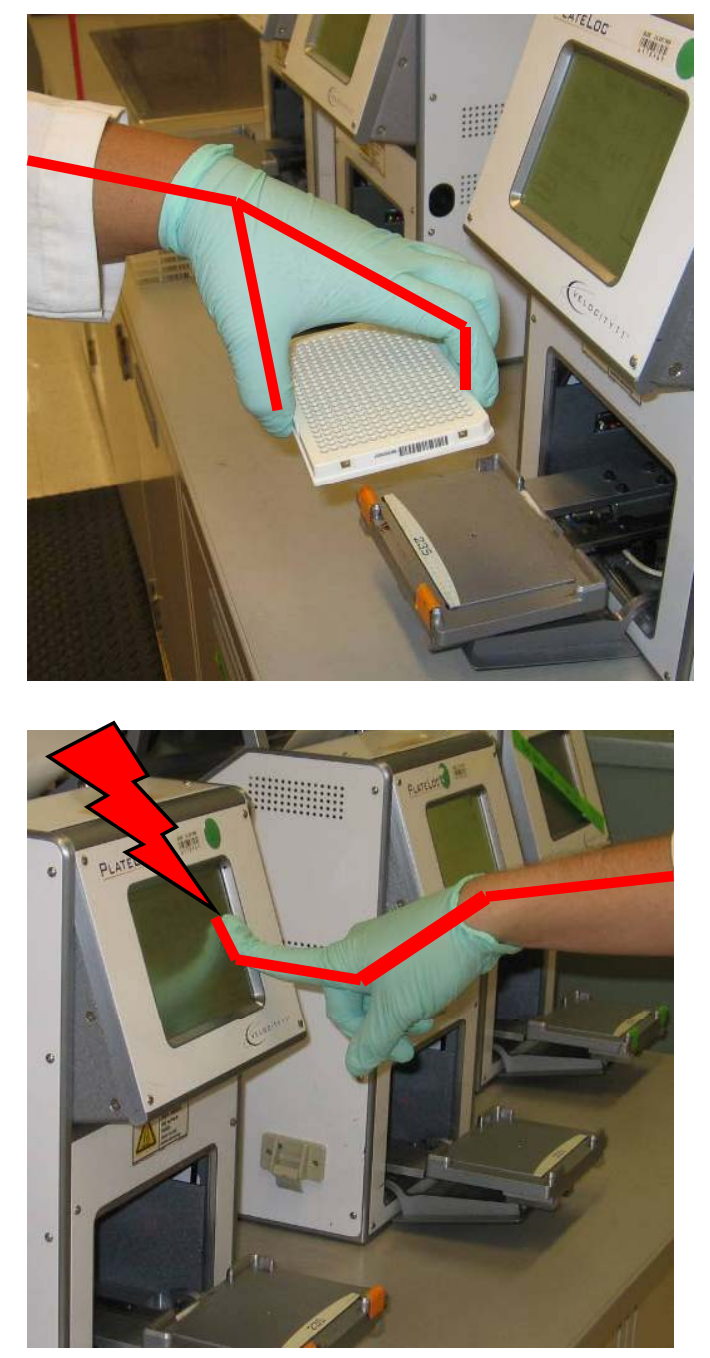

Before

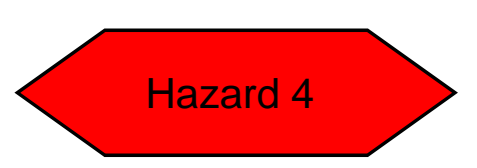

Avoid using a "claw grip. Use two hands to load and unload plate onto sealer. Press the start button lightly
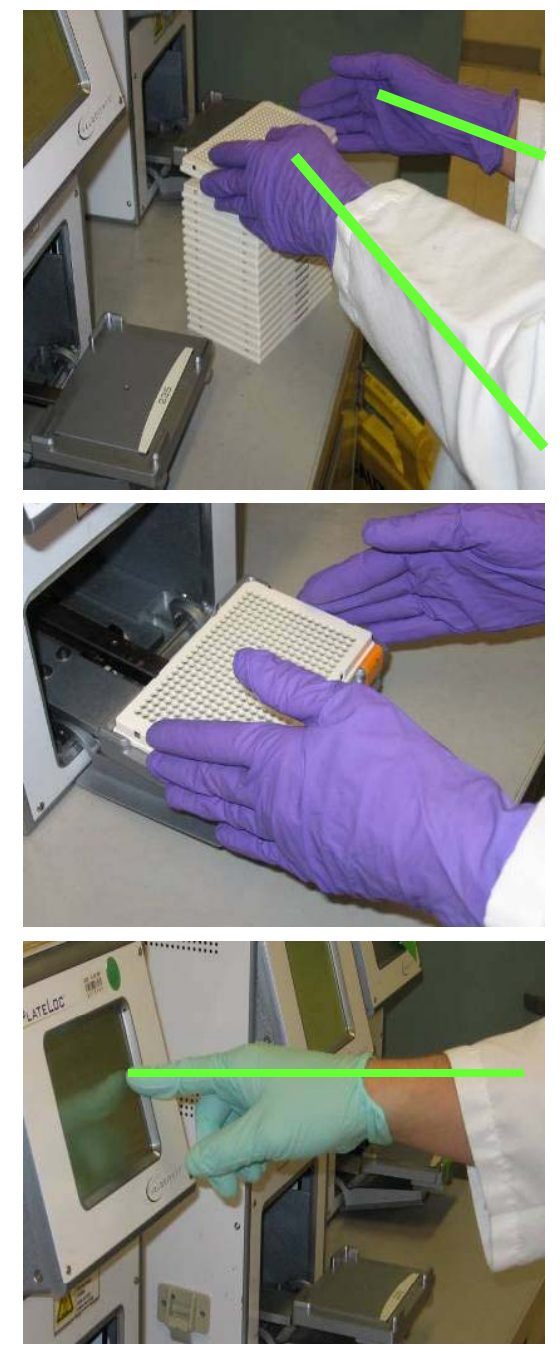

After 


\section{5) Required Practices}

- Example Slides 


\section{J厂5 \\ Dispense Visual Check}

Perform a visual check of every $4^{\text {th }}$ plate after Centrifugation. Use two hands to hold plate, do not raise above eye level.

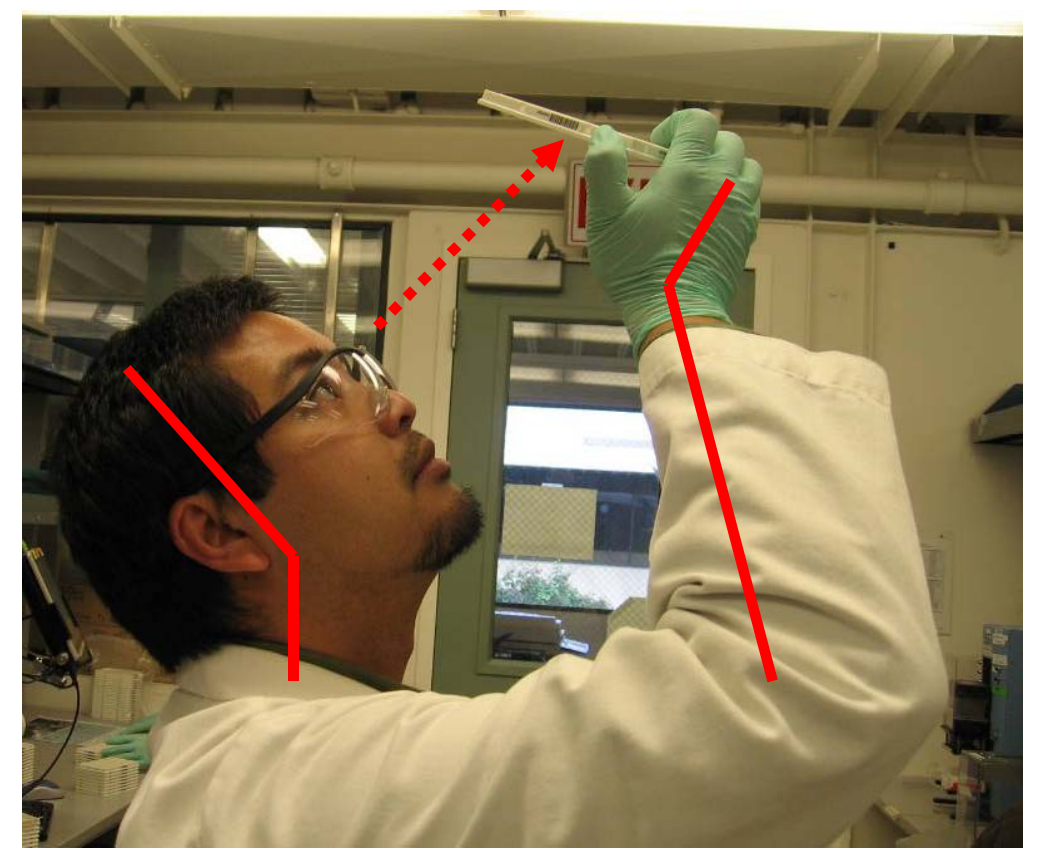

Before

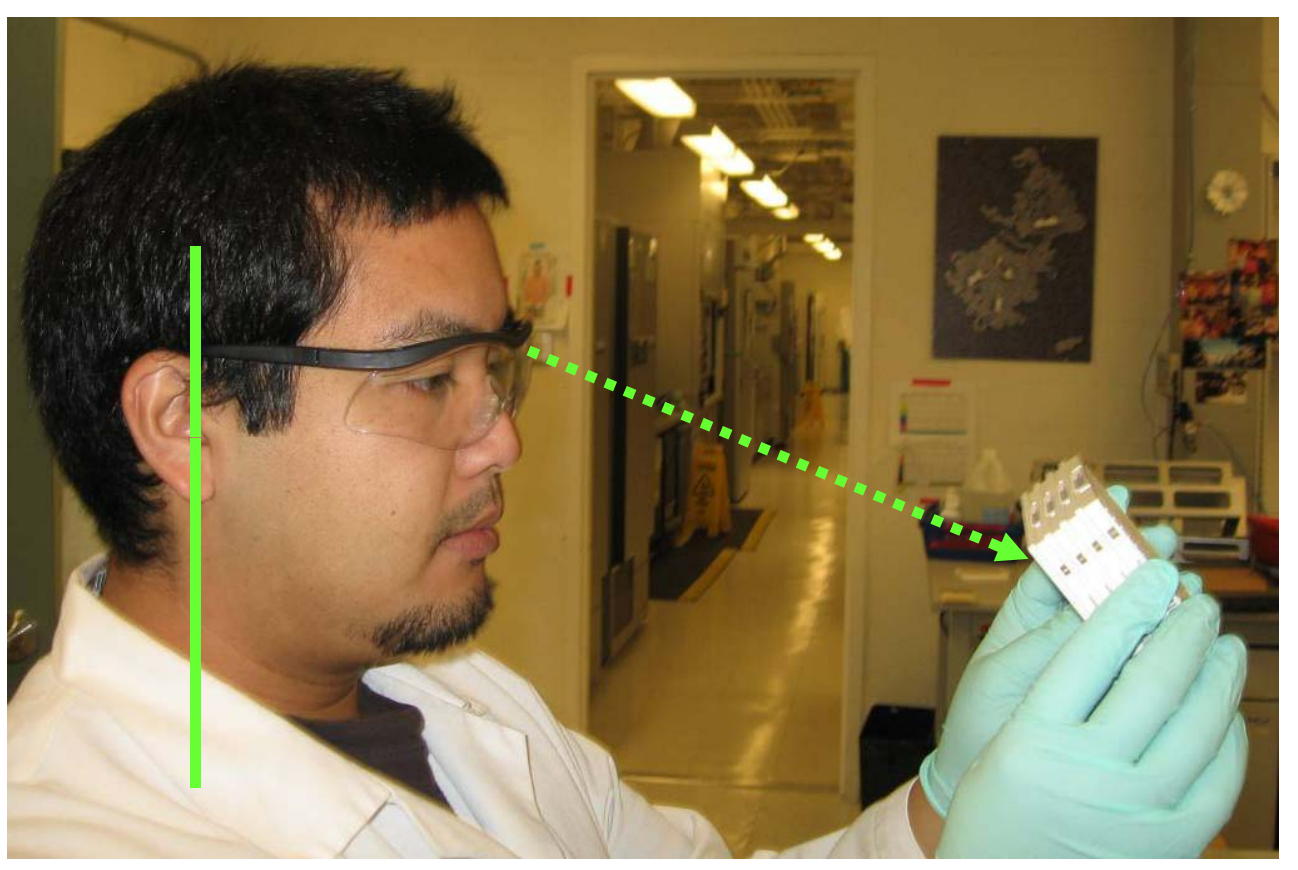

After 


\section{How to Remove Tight Caps Off Bottles}

Use a pair of slip joint pliers to remove the cap. Do not try to open reagent bottles with your hands.

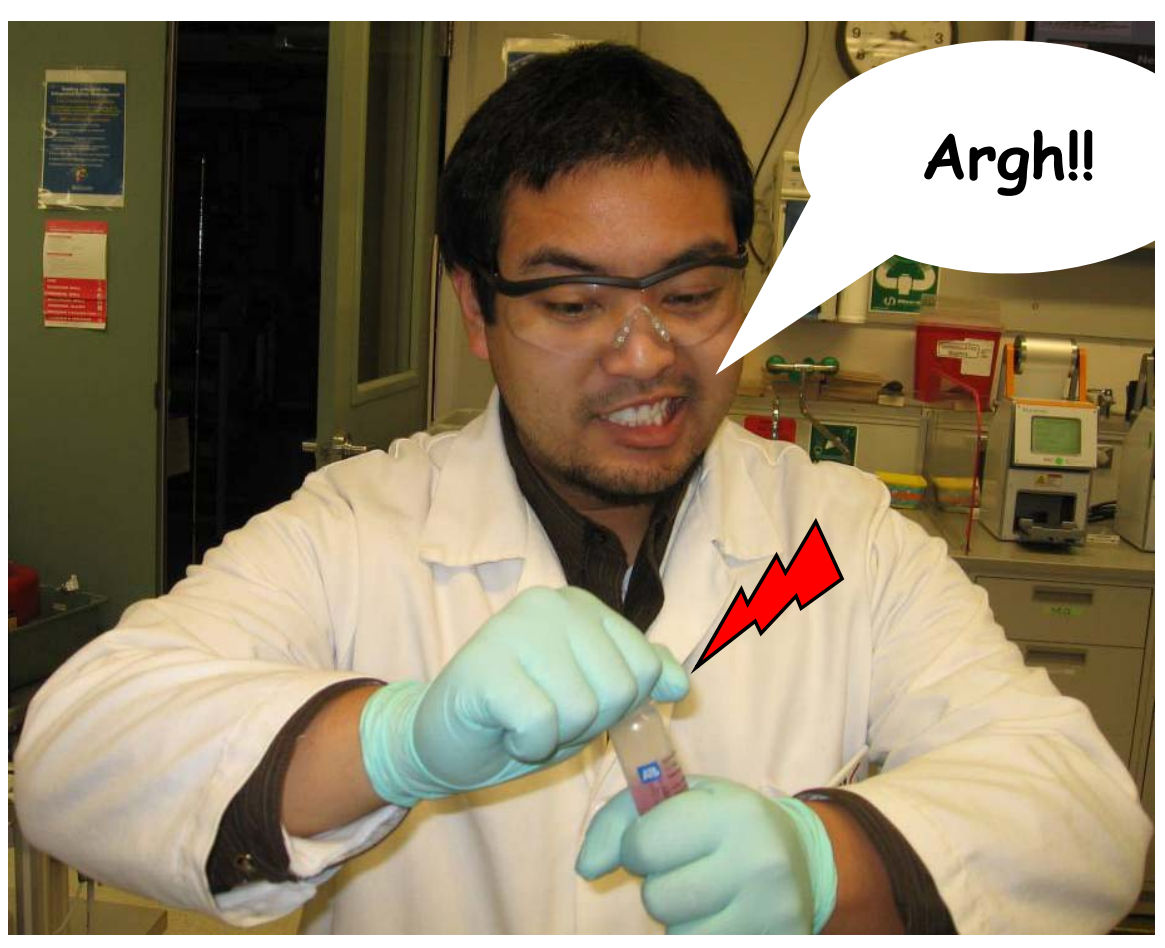

Before

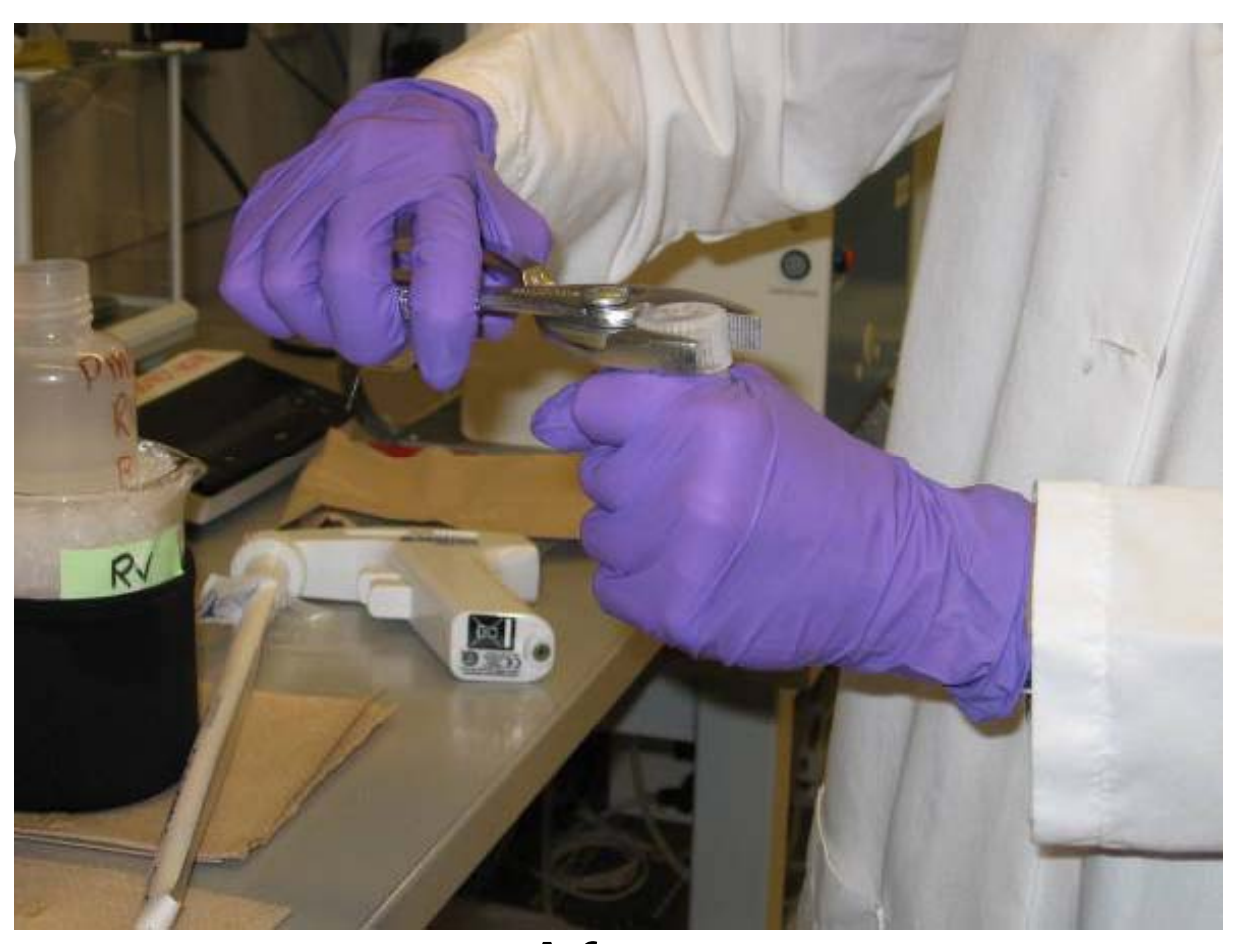

After 


\section{How to load and unload the Cybio}

Adjust the table to a height that is good for you. Avoid raising your elbow above shoulder level and utilizing awkward hand postures. Load no more than 16 plates at a time.

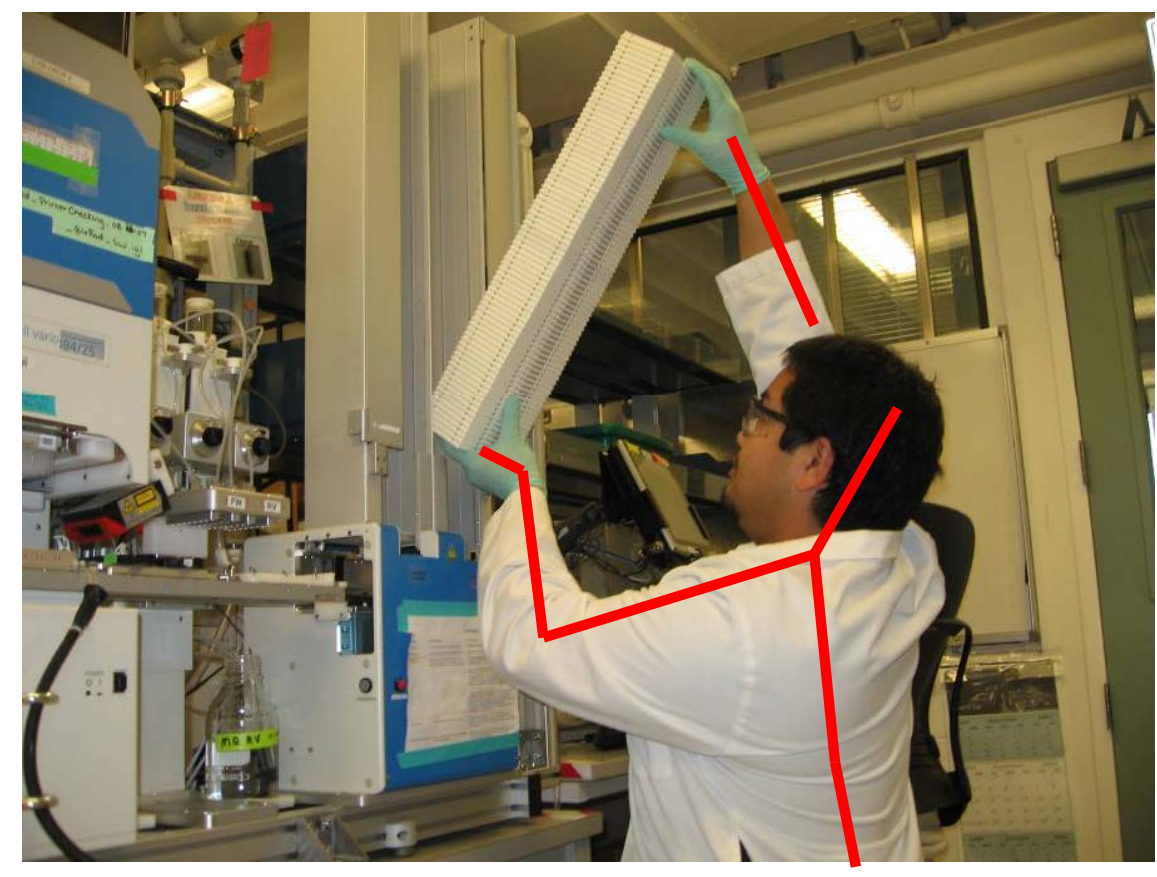

Before

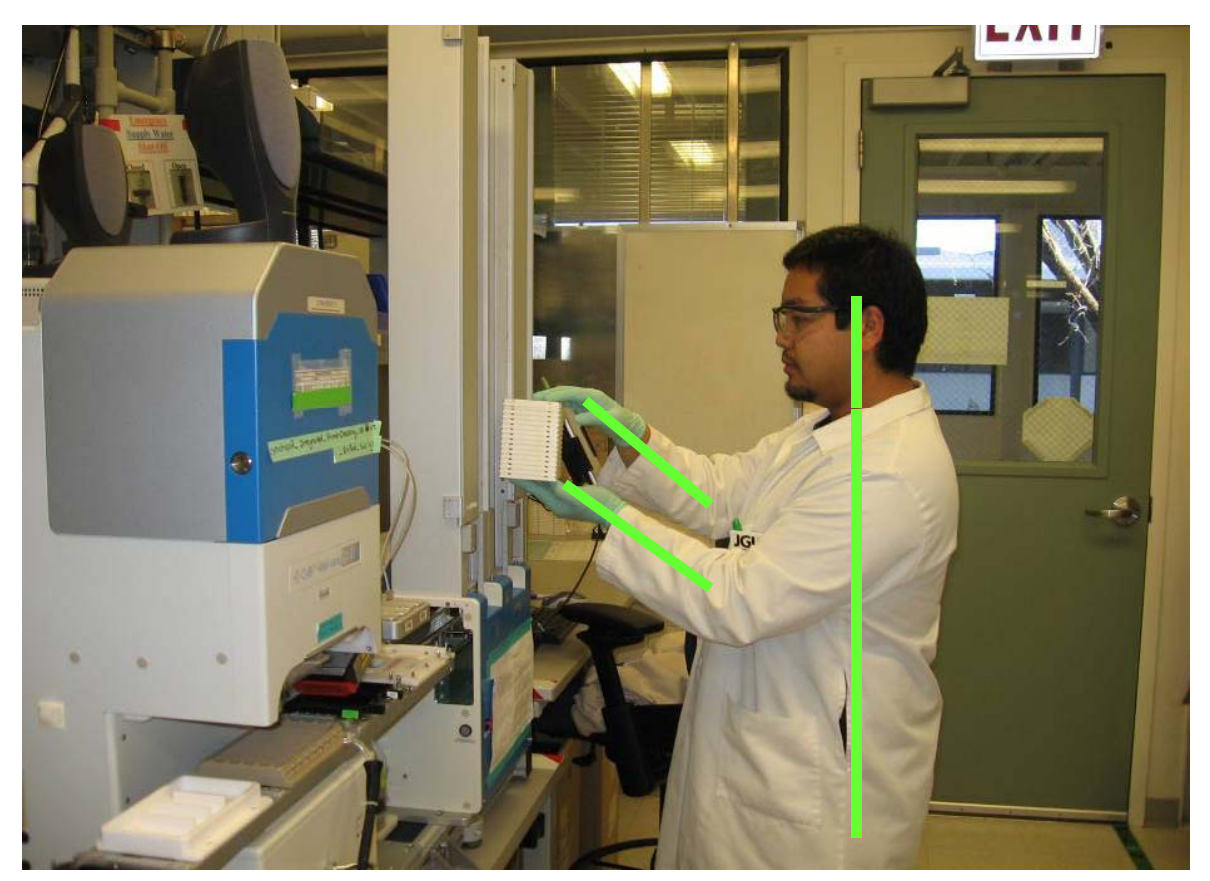

After 


\section{Loading Reagents}

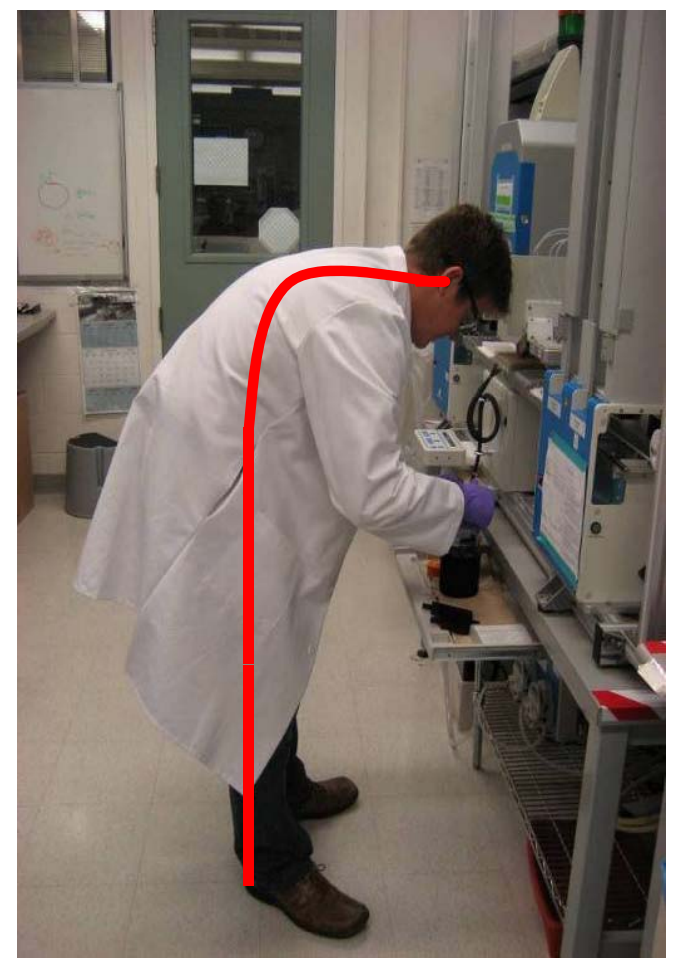

Before
Use a stool when loading reagents to avoid hunching over

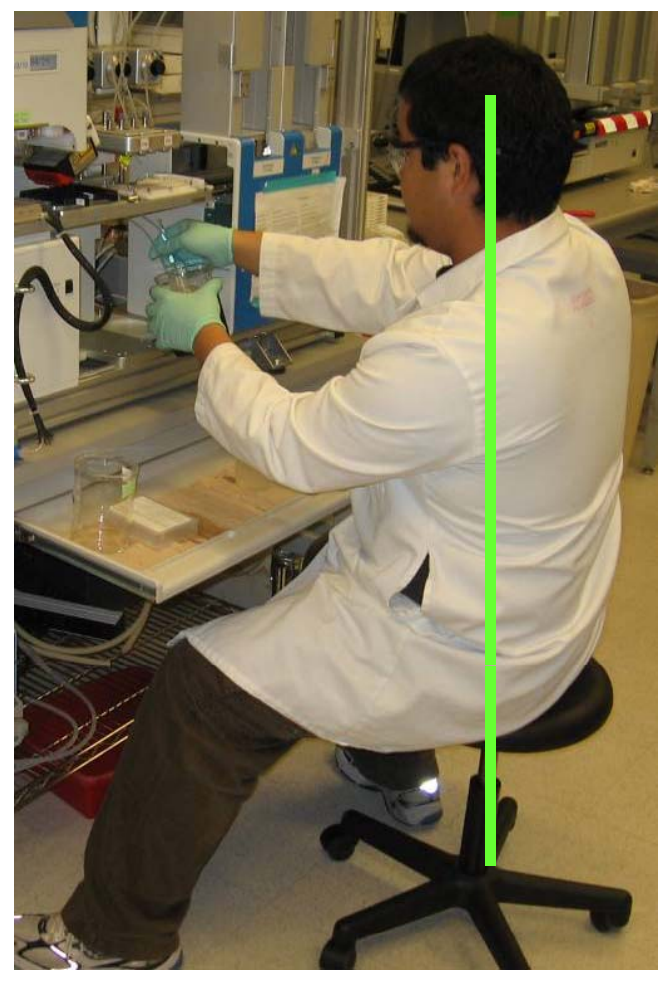

After 


\section{How to load Thermocyclers}

Load with both hands in a neutral position

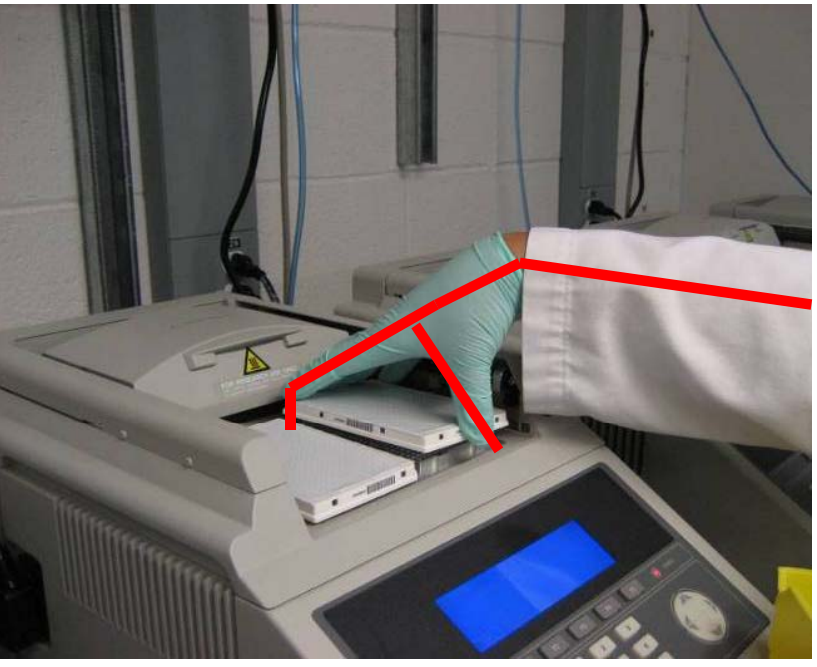
avoiding a claw-like grip as seen below in options $A$ and $B$

A

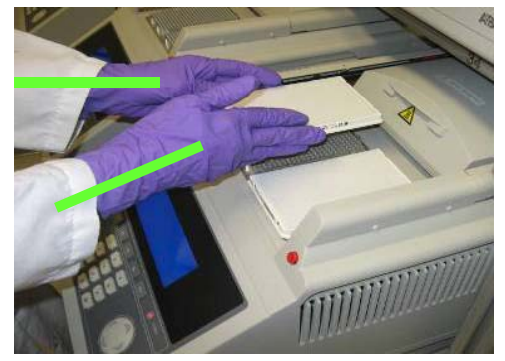

B

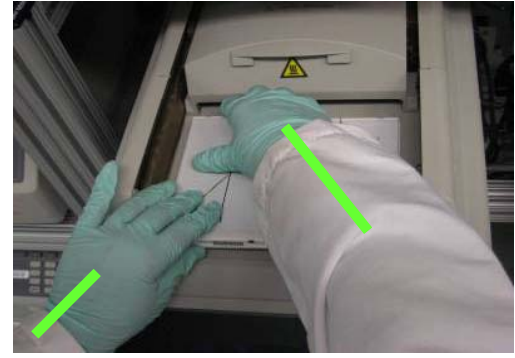

Load using the unloading tool, or drop the plate in place using a neutral position as seen below in options $C$ and $D$
C

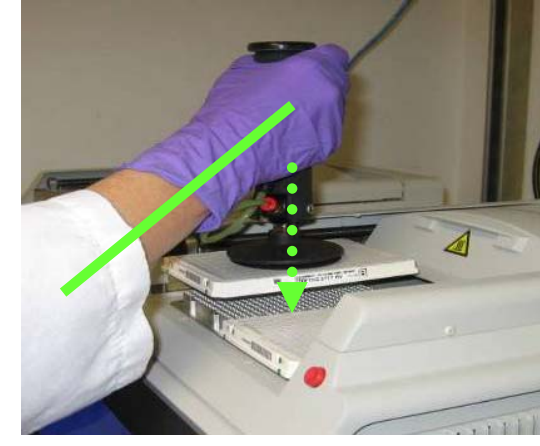

D

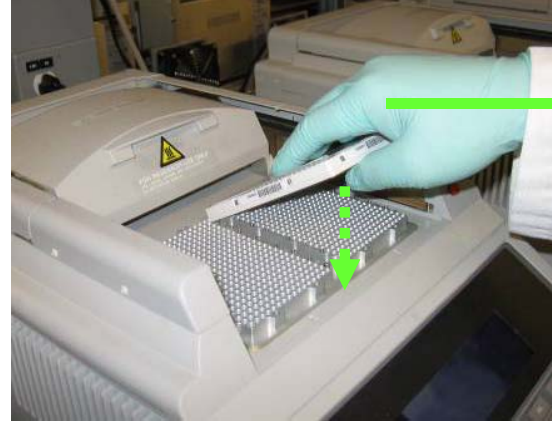

After 


\section{6) Operator Feedback: Safe Level of Plate Processing by Area}

\begin{tabular}{|c|c|c|c|c|c|c|c|c|c|c|}
\hline $\begin{array}{l}\text { Plating } \\
\text { (Bioassa } \\
\text { ys per } \\
\text { FTE) }\end{array}$ & $\begin{array}{l}\text { Pickin } \\
\text { g } \\
\text { (Plate } \\
\text { s per } \\
\text { FTE) }\end{array}$ & $\begin{array}{l}\text { OD } \\
\text { (Plat } \\
\text { es } \\
\text { per } \\
\text { FTE) }\end{array}$ & $\begin{array}{l}\text { Fos } \\
\text { Inoc } \\
\text { (Plat } \\
\text { es } \\
\text { per } \\
\text { FTE) }\end{array}$ & $\begin{array}{l}\text { Rca } \\
\text { (Plates } \\
\text { per FTE) }\end{array}$ & $\begin{array}{l}\text { Gels } \\
\text { (Gel } \\
\text { per } \\
\text { FTE) }\end{array}$ & $\begin{array}{l}\text { CHEM } \\
\text { (Plates } \\
\text { per } \\
\text { FTE) }\end{array}$ & $\begin{array}{l}\text { BET } \\
\text { (Plat } \\
\text { es } \\
\text { per } \\
\text { FTE) }\end{array}$ & $\begin{array}{l}\text { Caps } \\
\text { (Loaded } \\
\text { Plates } \\
\text { per FTE) }\end{array}$ & $\begin{array}{l}\text { Caps } \\
\text { (Debased } \\
\text { Plates } \\
\text { per FTE) }\end{array}$ & $\begin{array}{l}\text { Caps } \\
\text { (Machine } \\
\text { S } \\
\text { Running } \\
36 \mathrm{~cm} \text { ) }\end{array}$ \\
\hline 40 & 104 & 104 & 40 & 104 & 0.8 & 208 & 208 & 208 & 208 & 34 \\
\hline 38 & 100 & 100 & 40 & 100 & 0.8 & 200 & 200 & 200 & 200 & 32 \\
\hline 46 & 120 & 120 & 40 & 120 & 1.0 & 240 & 240 & 240 & 240 & 39 \\
\hline 31 & 80 & 80 & 80 & 80 & 0.6 & 160 & 160 & 160 & 160 & 26 \\
\hline 16 & 42 & 42 & 42 & 42 & 0.3 & 84 & 84 & 84 & 84 & 14 \\
\hline 16 & 42 & 42 & 42 & 42 & 1.0 & 84 & 84 & 84 & 84 & 14 \\
\hline 32 & 84 & 84 & 84 & 84 & 0.7 & 168 & 168 & 168 & 168 & 27 \\
\hline 15 & 40 & 40 & 40 & 40 & 0.3 & 80 & 80 & 80 & 80 & 13 \\
\hline 12 & 30 & 30 & 30 & 30 & 0.2 & 60 & 60 & 60 & 60 & 10 \\
\hline 15 & 40 & 40 & 40 & 40 & 0.3 & 80 & 80 & 80 & 80 & 13 \\
\hline
\end{tabular}




\section{Additional Changes}

- Organizational Structure changes

- Throughput model

- Partner labs commitment from medical

- Streamlined return to work program

- Early Intervention Program

- Full Time Ergonomics Support (3 ergonomists)

- Monitoring program

- Onsite Nurse on Tues, Thurs PM

- Process Change Notification

- Observation Program

- Structured process for rotation on the production floor

- Ergo points 


\section{Clarifying Organizational Roles and Responsibilities}

\begin{tabular}{|c|c|}
\hline Role & Responsibilities \\
\hline Production Coordinator & $\begin{array}{l}\text { - Managing and coordinating all aspects of the sanger line from Plating } \\
\text { through } A B I \\
\text { - Responsible for change management on the line } \\
\text { - Throughput, scheduling, staffing } \\
\text { - Supervising the Technical and Administrative supervisors }\end{array}$ \\
\hline Technical Lead & $\begin{array}{l}\text { - Single point of contact on designated shift to make all technical } \\
\text { decisions } \\
\text { - Shift meetings } \\
\text { - Anything associated with processing samples } \\
\text { - Interact with Supervisors on staffing problems, schedule changes } \\
\text { - Interact with Production coordinator on technical aspects of the } \\
\text { process } \\
\text {-Coordinating technical training }\end{array}$ \\
\hline Administrative Supervisor & $\begin{array}{l}\text { - Hire, performance management, time keeping, staff development } \\
\text { (1x1s) } \\
\text { - Anything wl HR } \\
\text { - Interact with Technical leads on time keeping, staffing problems and } \\
\text { schedule changes } \\
\text { - Interact with production coordinator on staffing needs } \\
\text { - Administrative training }\end{array}$ \\
\hline
\end{tabular}


- $20 \%$ overall reduction in throughput

FY2008 Production Numbers:

\begin{tabular}{|l|c|c|c|c|l|}
\hline & Q1 & Q2 & Q3 & Q4 & Total \\
\hline Sanger & 4.030 & 3.397 & 6.142 & 6.142 & 19.711 \\
\hline 454 & 3.164 & 6.603 & 7.398 & 7.398 & 24.563 \\
\hline & & & & & 44.274 \\
\hline
\end{tabular}




\section{CIS Partner Labs Commitment}

- HR

- Case review meeting

- Employee accommodations

- Medical

- Partner labs support for onsite Nurse

- Partner labs support for Early Intervention

- Partner labs now incorporate JGI ergonomist paperwork 


\section{JGI Early Intervention Process Chart: Monitoring, Observations}
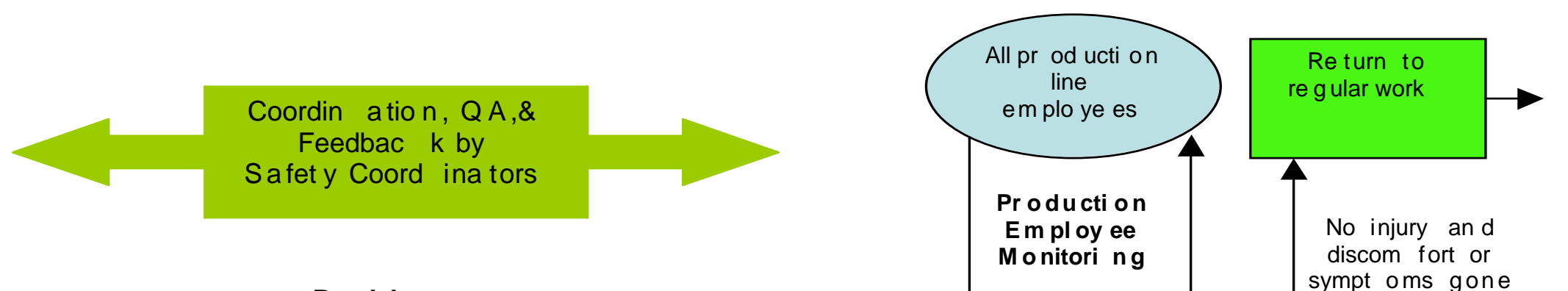

Proble $m$ Reco gnition

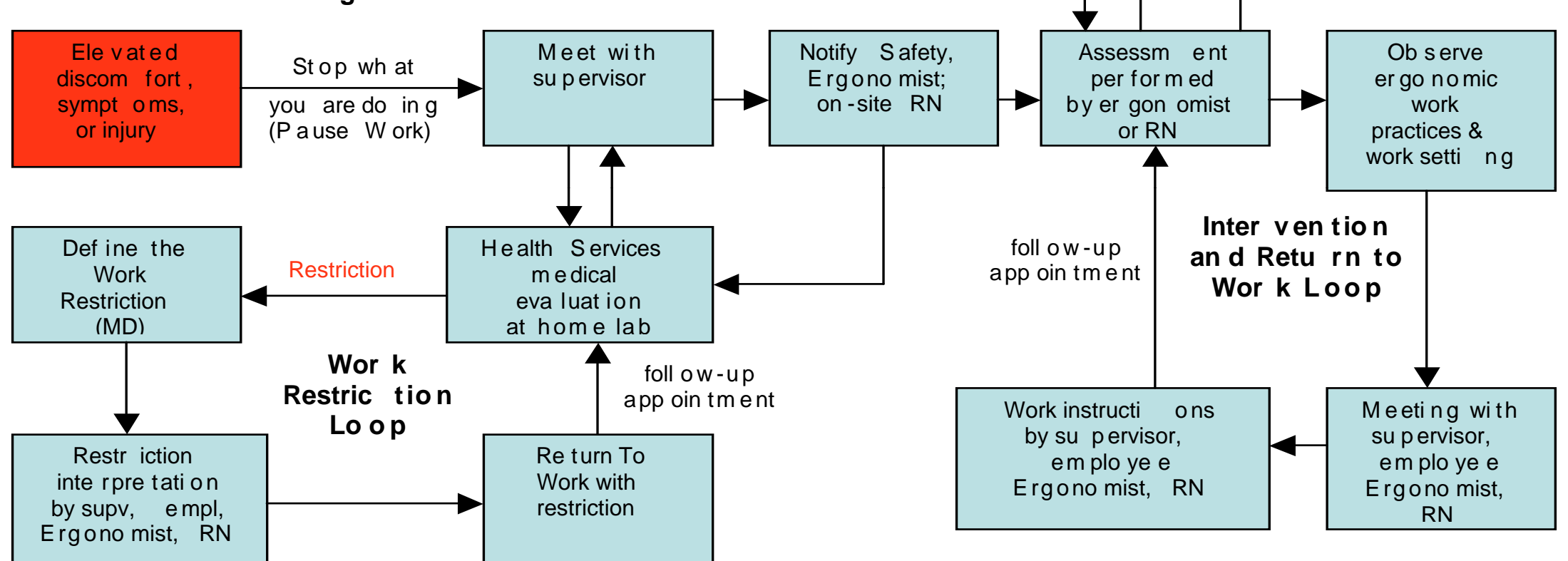




\section{Ergo Points}

- Supervisors want to know....

\section{"HOW MUCH IS TOO MUCH?"}

- Guidelines for schedulers

- What tasks can be scheduled together

- How many tasks can be conducted in one day

- No ergonomics risk tool sensitive for low force high repetition tasks like

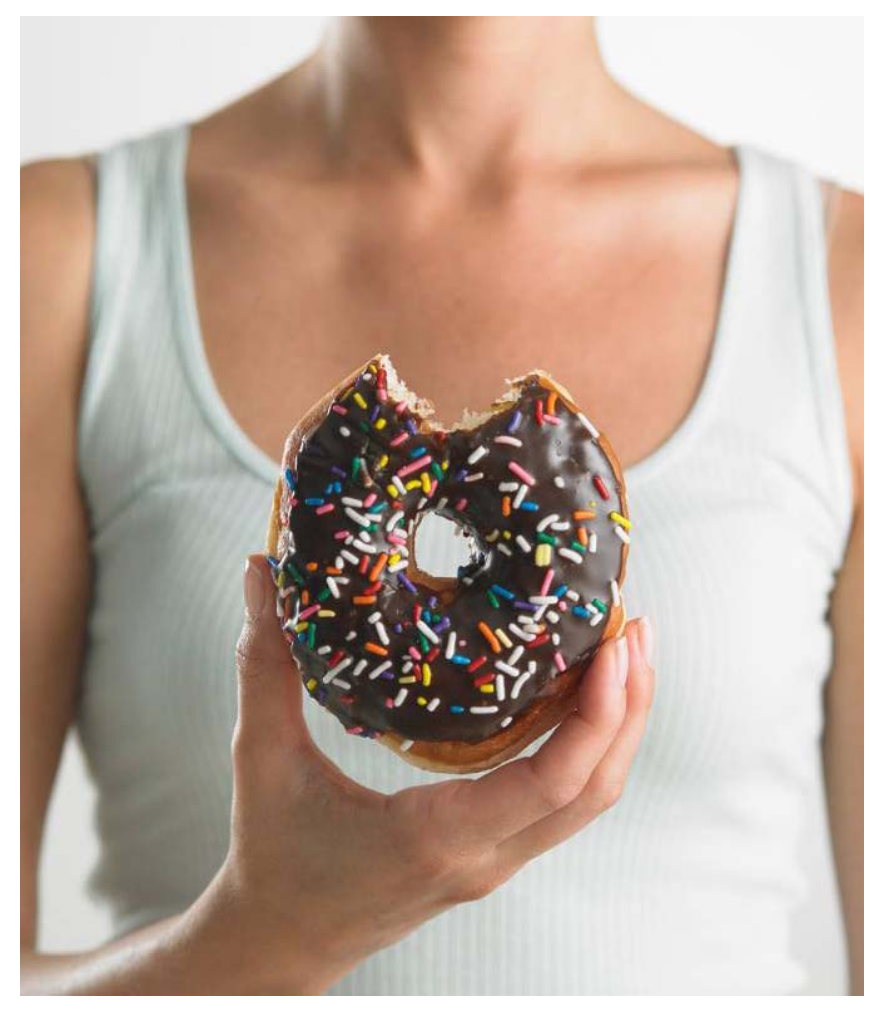
at JGI

GOAL: Reduce ergonomic risk caused by the combination of tasks assigned 


\section{'Ergo Points'}

\section{CWeightWatchers}

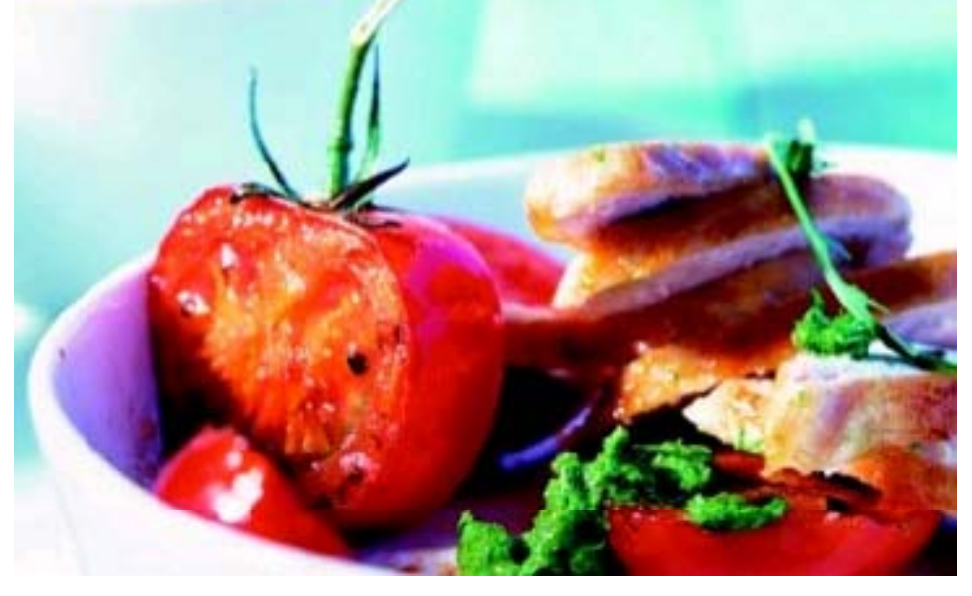




\section{Weight Watchers}

\begin{tabular}{|c|c|}
\hline Food & $\begin{array}{c}\text { Weight } \\
\text { Watchers } \\
\text { Points }\end{array}$ \\
\hline Medium Fries & 10 \\
\hline Cheeseiourger & 7 \\
\hline Big Mac & 14 \\
\hline Corn on Cob & 3 \\
\hline & 34 \\
\hline
\end{tabular}




\section{Ergo Points}

\begin{tabular}{|l|c|}
\hline \multicolumn{1}{|c|}{ Task } & Ergo Points \\
\hline Fanditing bio-assay trays & $\mathbf{2 0}$ \\
\hline $\begin{array}{l}\text { Loading/Unloading Stackers } \\
\text { (Top Loading) }\end{array}$ & 16 \\
\hline $\begin{array}{l}\text { Loading/Unloading Stackers } \\
\text { (Front Loading) }\end{array}$ & 5 \\
\hline $\begin{array}{l}\text { Unsealing Heat Seals } \\
\text { DAlLY TOTAL }\end{array}$ & $\mathbf{1 8}$ \\
\hline
\end{tabular}


Job / Task: RCA: Peeling Foil Seals

SI Score Interpretation

$$
\text { Date: } 7 / 27 / 2006
$$

Analyst: Christine Naca, Ira Janowitz

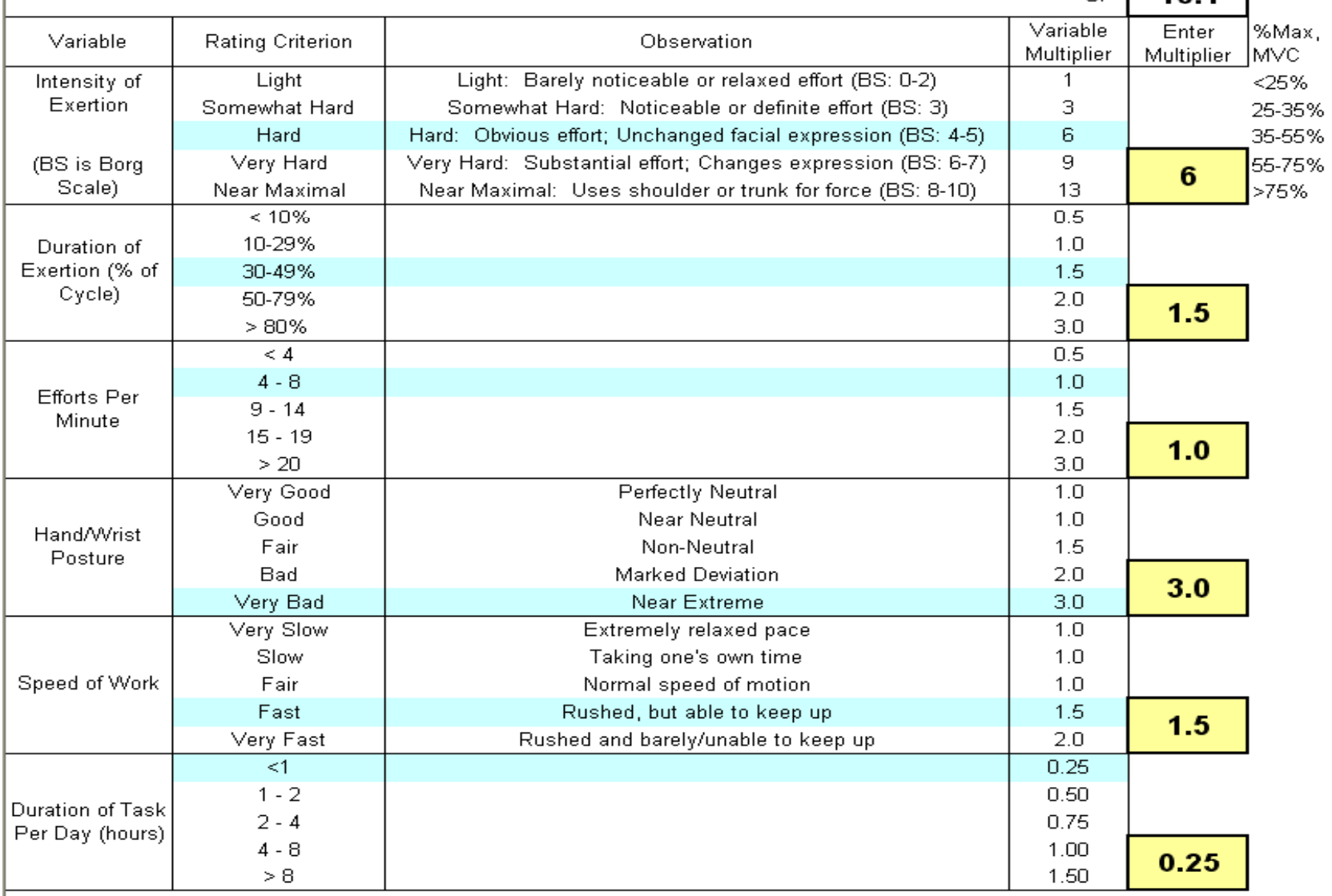

Note: This worksheet was adapted and interpreted

by the USF investigators. No warranty is offered.

Reference: J. Steven Moore \& Arun Garg, Thomas E. Bernard and Robert B. Walton

The Strain Index: A Proposed Method to University of South Florida

Analyze Jobs For Risk of Distal Upper College of Public Health

Extremity Disorders; Am. Ind. Hyg. Assoc. Tampa FL 33612-3805 (813) 974-6629

J. $56: 443-458(1995)$

Partial support from

UAW-Ford NJCHS

tbernard@hsc.usf.edu and rwalton@ihsc.usf.edu

Ford Motor Company

v2.21/11/01@2001 Thomas E. Bernard

For updates, see Stone Wheels at Www.hsc.usf.eduj tbernard

No Warranty: Expressed or Implied.

\section{Strain Index assumes conducting same task all day long.}




\section{Development of 'Ergo Points'}

- Task-specific to JGI production jobs

- First 20 tasks based on synthesis of Strain Index, expert opinion, and anonymous employee discomfort ratings to 'calibrate' data.

- Statistical tests for agreement

- Apply to other production tasks 


\section{Using Ergo Points Today}

- Supervisors use it as a 'sandbox' to test out various combinations and quantities of tasks

- Used to validate new processes and/or process changes i.e. new technologies

Ergo Points continues to provide guidelines to identify ergonomic risk exposure 


\section{Before we could start back up...}

$\square$ Area fully documented, required practices in place

$\square$ Hazard solutions need to be reasonable and meet operator expectations

$\square$ You must feel safe with the throughput levels and the work pace

$\square$ Operators fully trained to work in the area

$\square$ Clear expectations for operators and supervisors

$\square$ Process Change Notification in place

$\square$ Communication Model in place

$\square$ Early intervention in place

$\square$ Return to work procedures in place

$\square$ Employee/Supervisor checklist in place (Check and Balance)

$\square$ Leadership in place 


\section{Plan: Ramping Back Up}

Double shifts

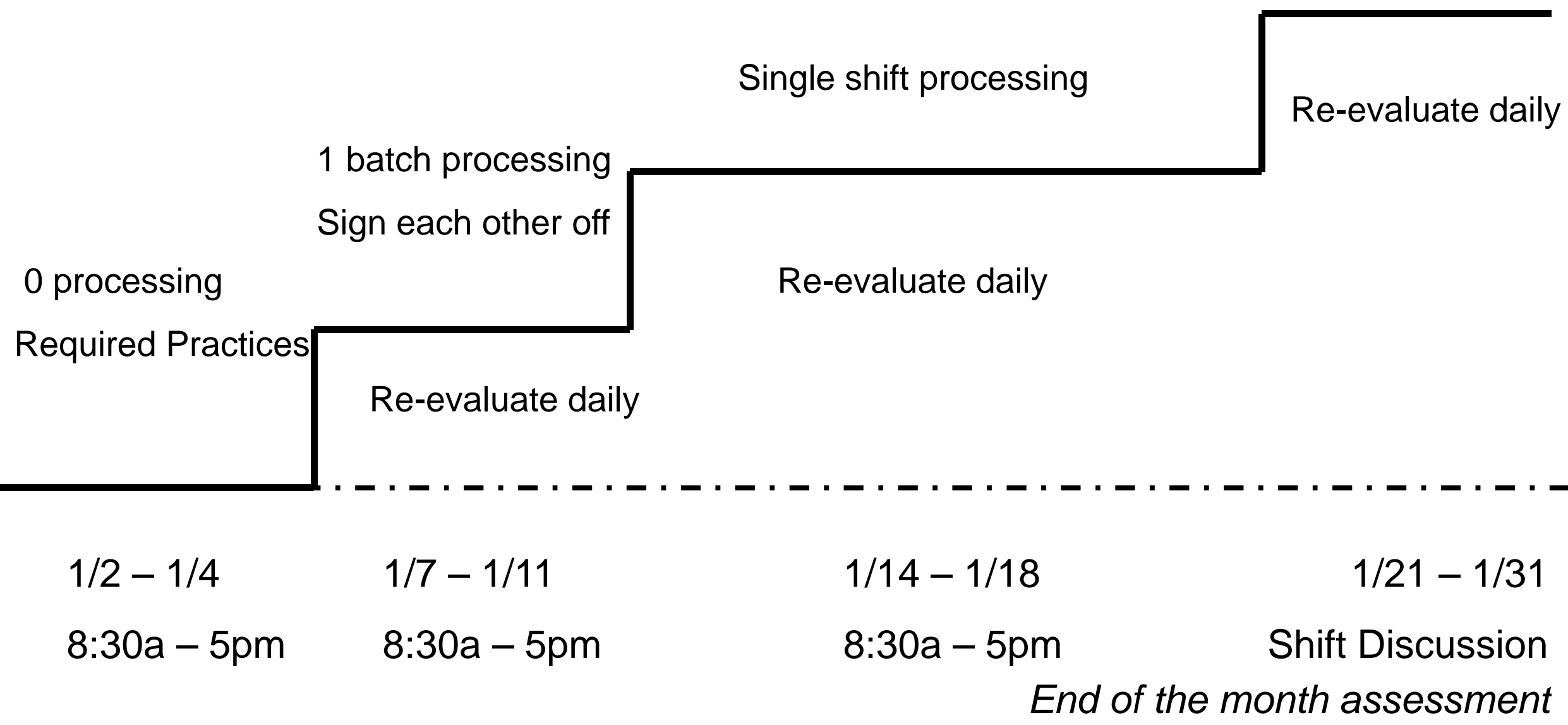




\section{FY2008 Goals}

- Back to processing 4 batches/day, which is an overall $20 \%$ reduction of processing plates

- Administrative/Engineering modifications to the line

- Incorporating next generation technologies into production 


\section{Vying to retain the Ergo Cup 2008}

- The JGI Production Department competed with two new innovations.

- $\quad$ Kept Up Employee Morale

\section{"Base Off"}

\section{Before}

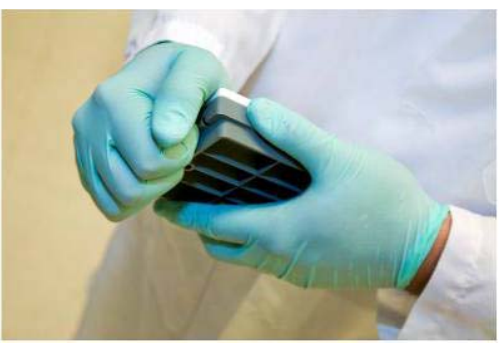

After

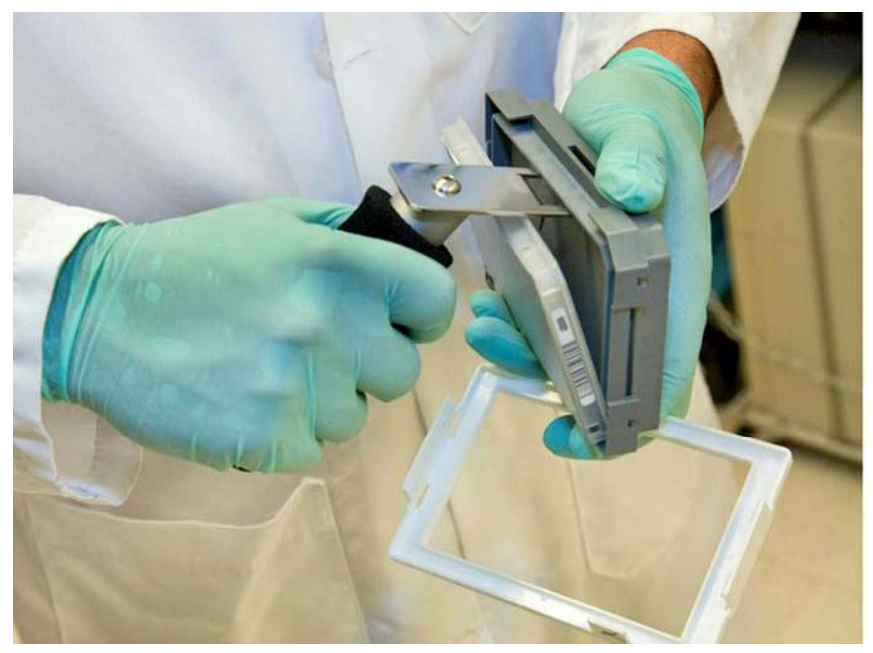

"It's A Peeling"
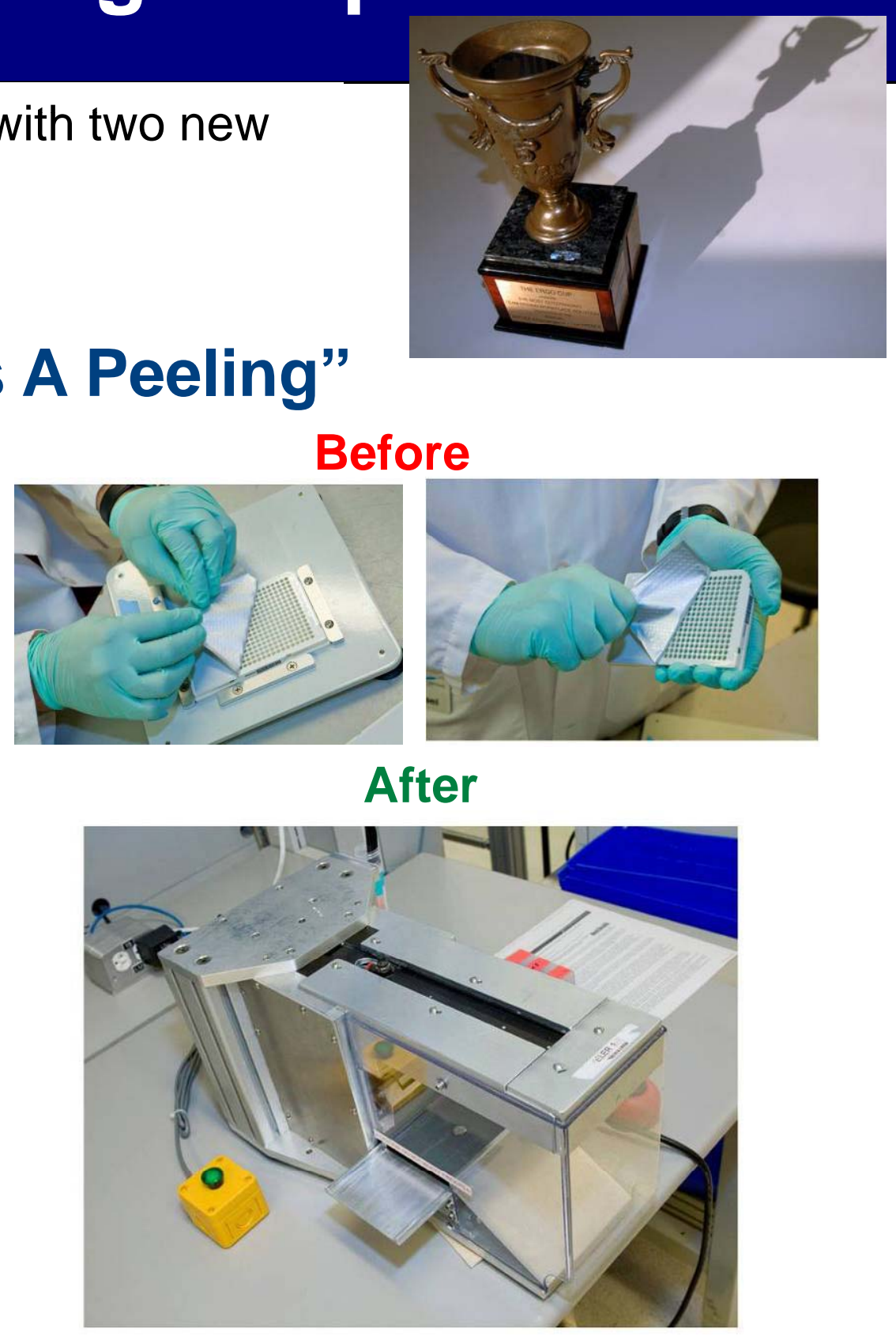


\section{What we learned?}

- No 'silver bullet'

- Ergonomics is a team effort

- Required an organizational solution

- Change in culture (productivity vs safety)

- Staff ownership

- Accountability

- Support from Medical, HR, Safety, LLNL and LBL

- Takes time and patience to change

- Change is difficult!! 


\section{CIS Commitment To Zero Injuries}

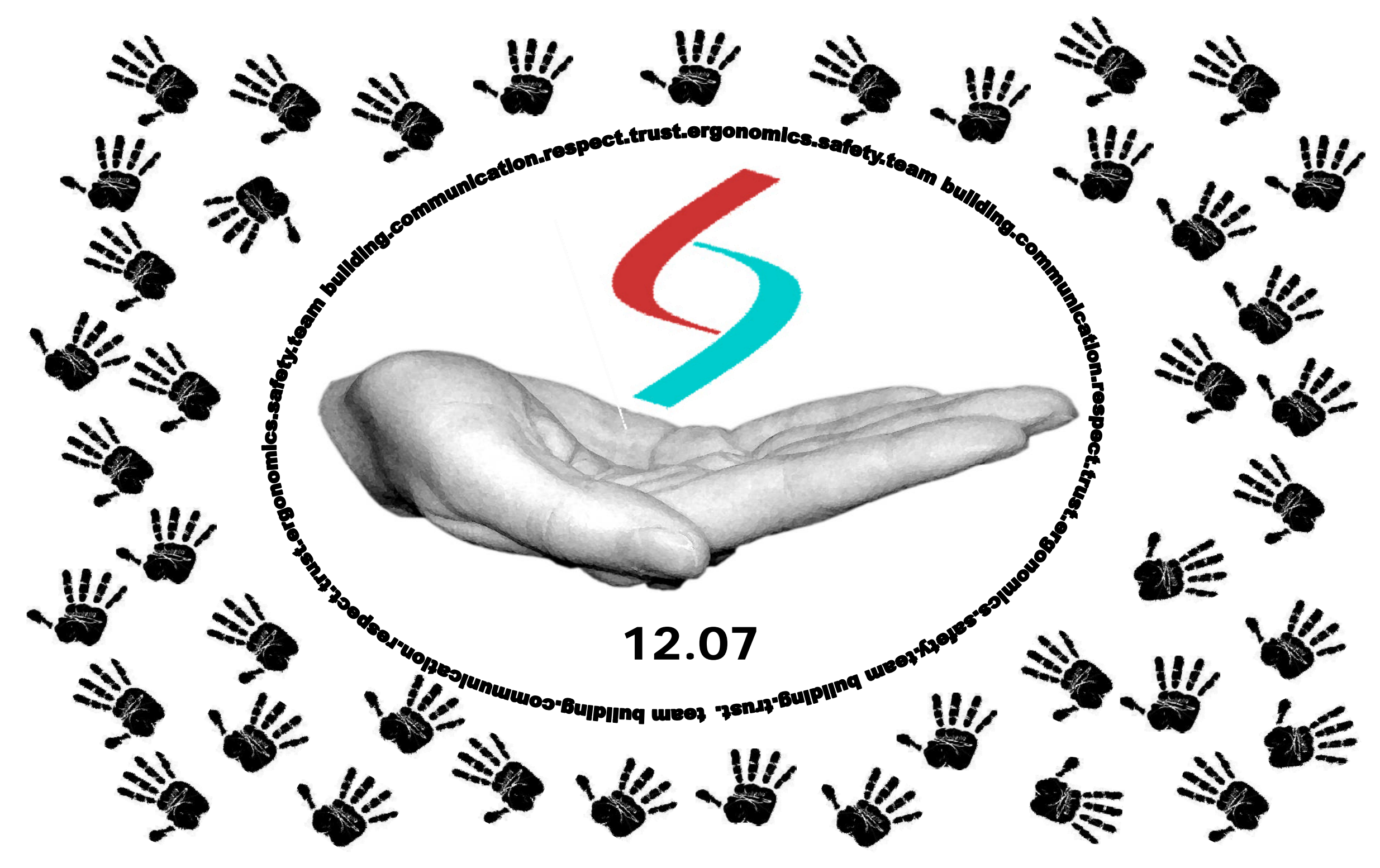




\section{Engineering designs and solutions}

\section{Early intervention}

Targets employees

with discomfort

Includes bi-weekly

review meeting

Proactive Ergonomics Risk Assessments/Evaluations

Labs and offices

Monitoring

Walk-abouts

Comfort surveys

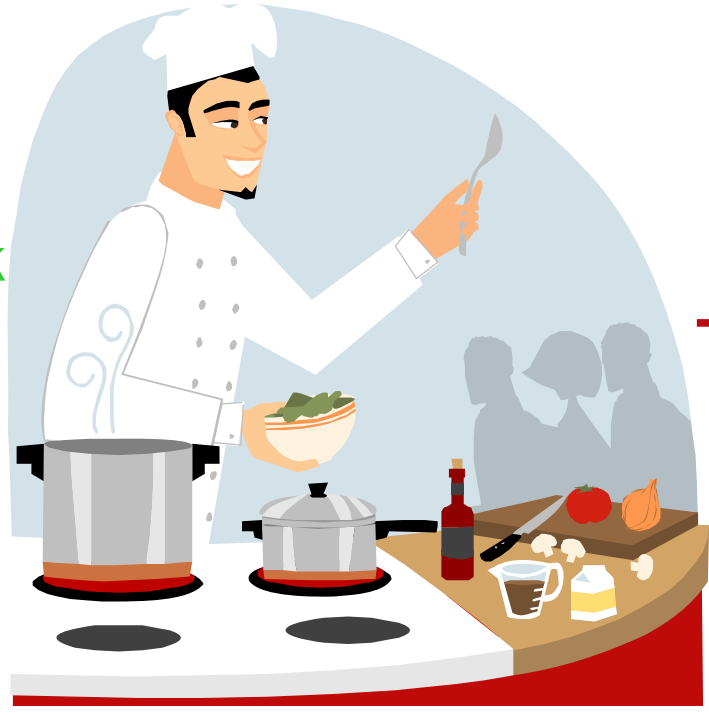

Ergonomics Working Group

Promotion

Awareness

Communication

Training/education

Risk targeted courses

Stretch break programs

Potty training

Website resources

\section{Relaxation/}

Rejuvenation Room

\section{Ergonomics Demo Room}

Work tool and practices

Ergo Points

Required Practices 

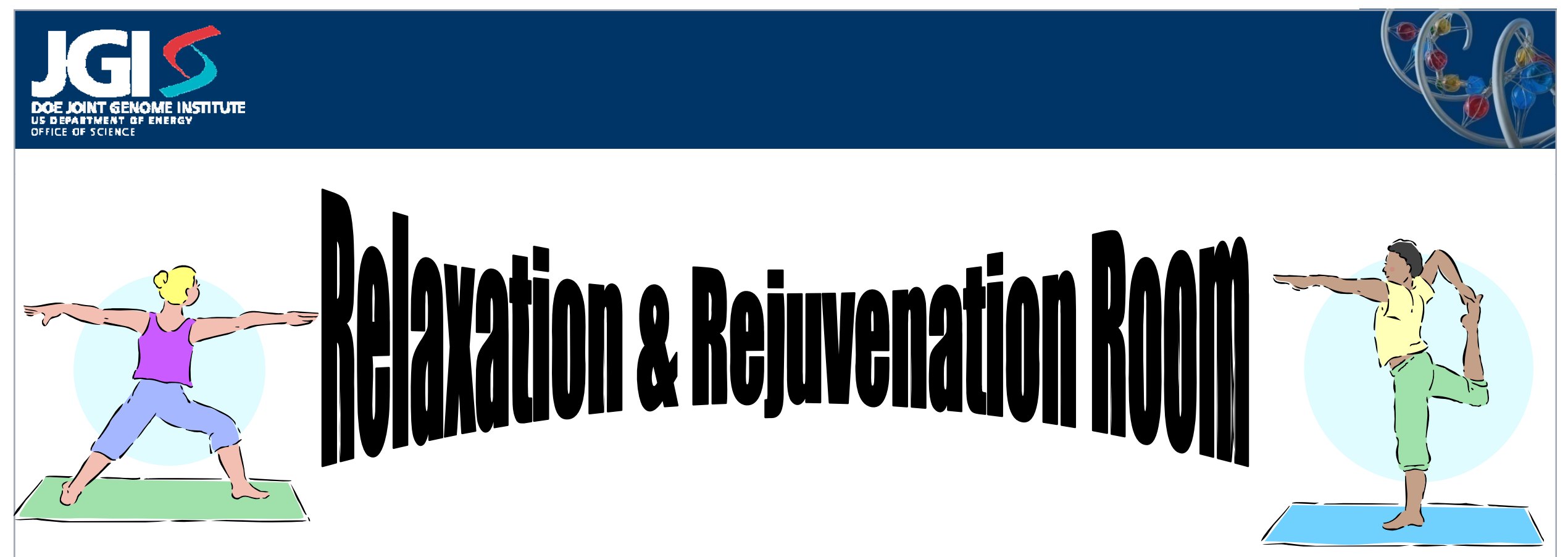

Room 350 in Building 310 is your place to stretch, ice down, and take pilates classes. 350 is reserved exclusively for stretching, so stop by anytime during work hours to stretch or ice.

Come try the

- foam mats

- foam rollers

- massage tools

- hot and cold packs
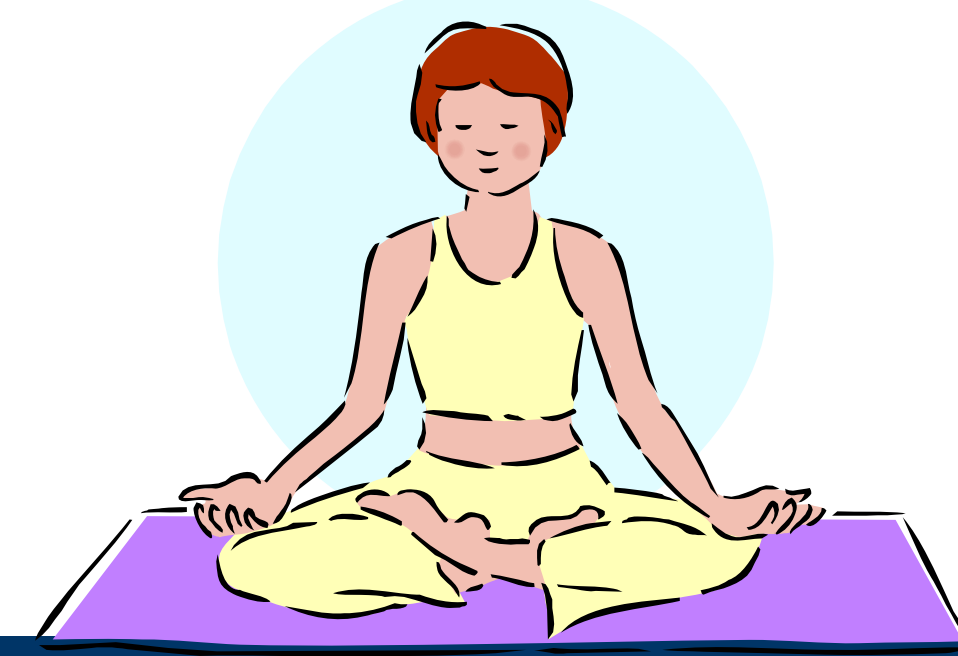

Pilates classes in 350 with Wendy Schackwitz Mon 12:00 - 12:30 Wed $\quad 4: 30-5: 30$

Fri $\quad 12: 00-12: 30$ 

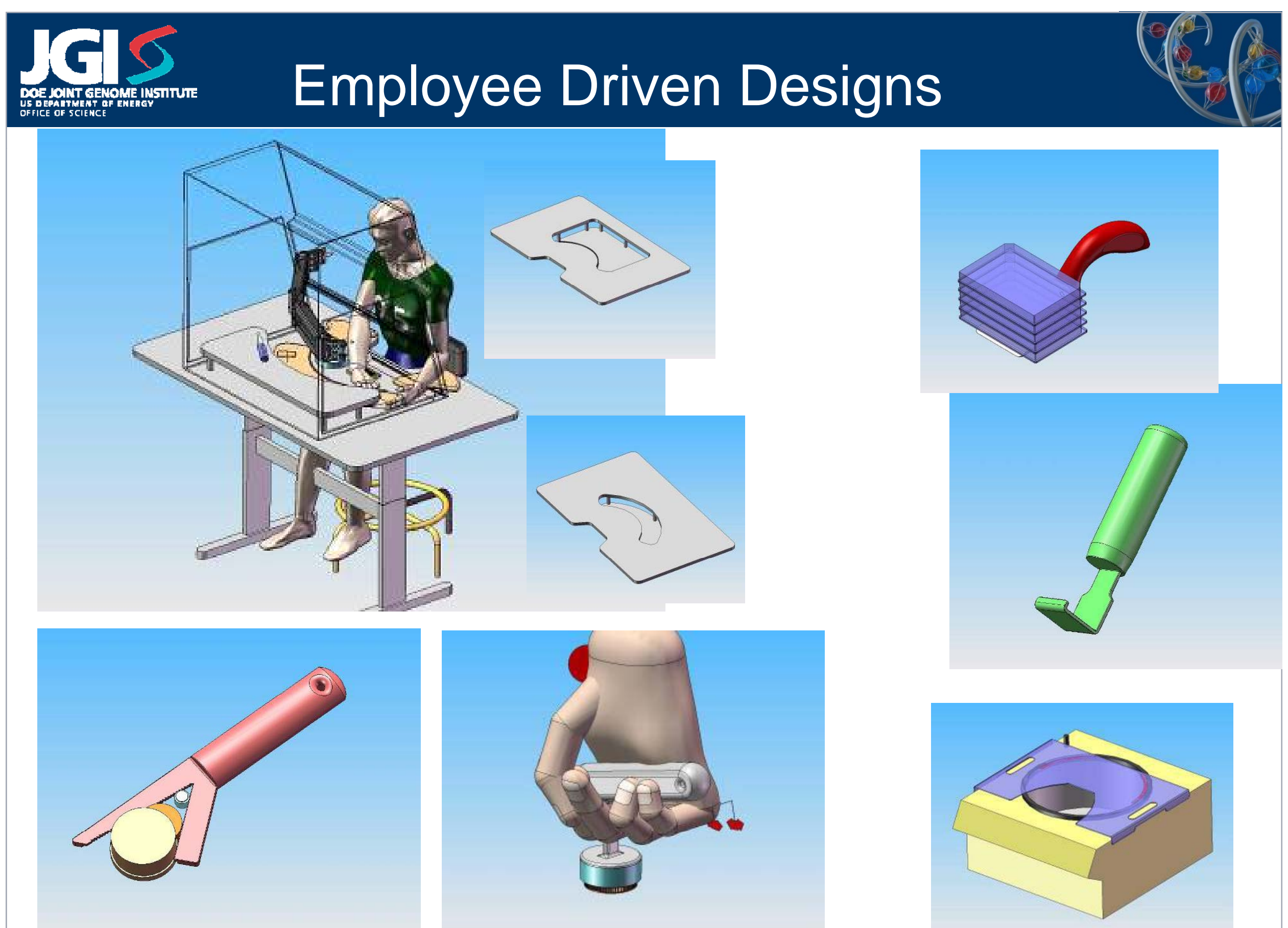


\section{Continuous Improvement}

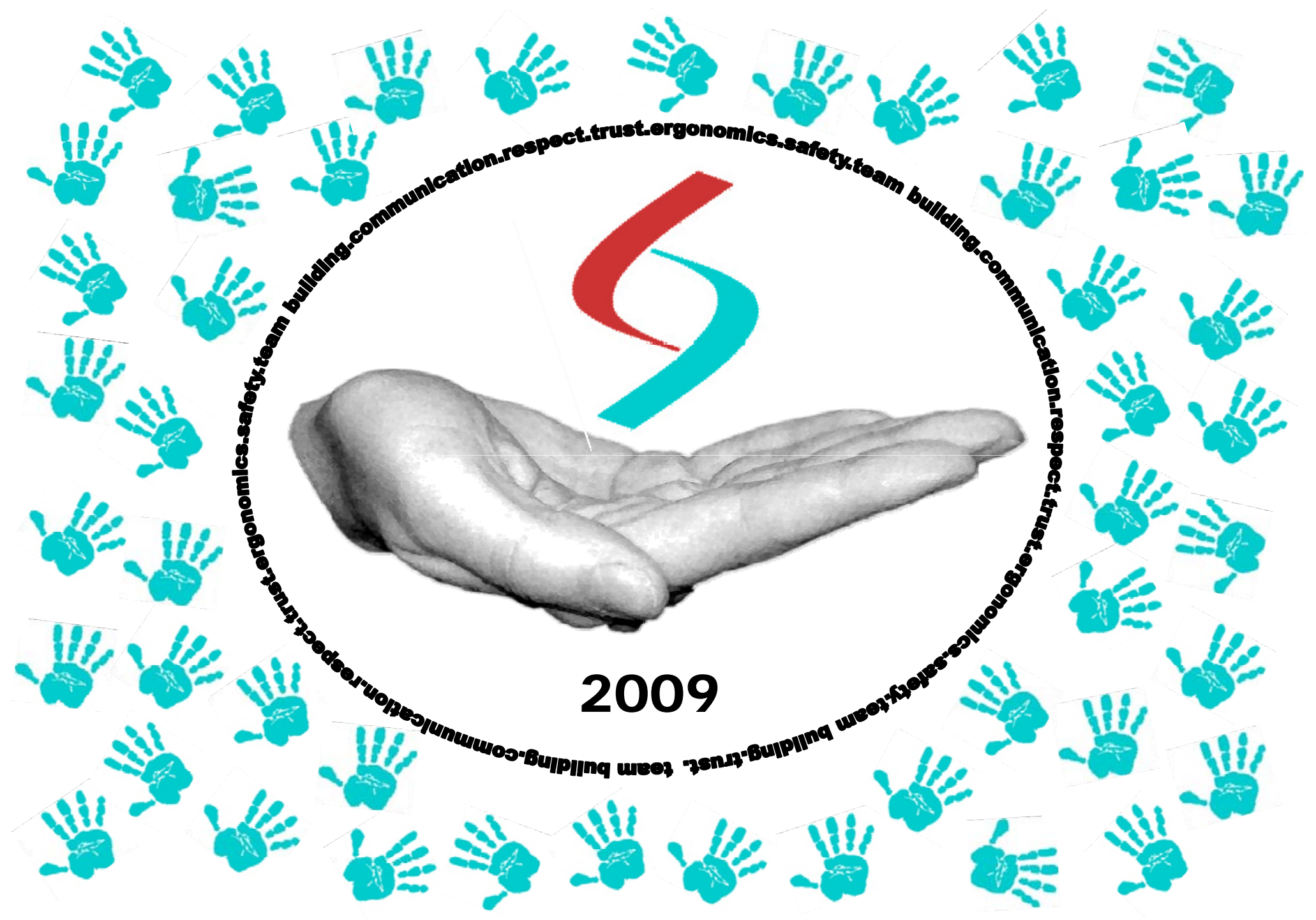

Recommitment to Zero Injuries and Safety 

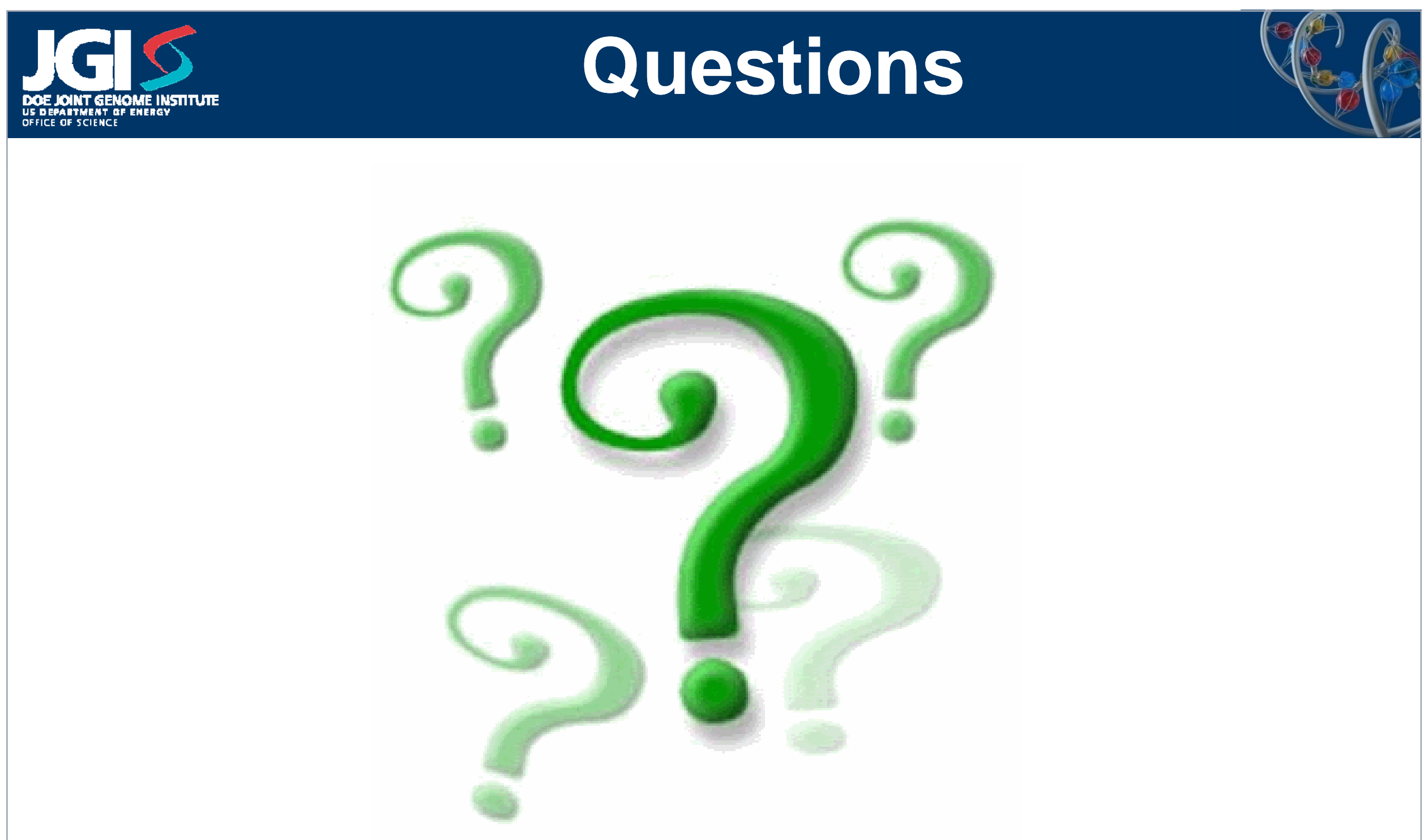

Acknowledgements:

Susan Lucas, Melanie Alexandre, Ira Janowitz, Andy Imada, Stephen Franaszek, Debra Rosett, Simon Roberts, Dr. David Rempel

This work was performed under the auspices of the US Department of Energy's Office of Science, Biological and Environmental Research Program, and by the University of California, Lawrence Berkeley National Laboratory under contract No. DE-AC02-05CH11231, Lawrence Livermore National Laboratory under Contract No. DE-AC52-07NA27344, and Los Alamos National Laboratory under contract No. DE-AC02-06NA25396. 


\section{'Ergo Points'}

More Detail 


\section{Identify Variables}

- Part I: Generate a Detailed Task List to indicate tasks performed in specific production areas.

- Group tasks that are conducted together by a single operator (multi-tasking impacts the risk).

- Part II: Collect a discomfort rating for each task (subjective metric)

\begin{tabular}{|c|c|}
\hline $\begin{array}{c}\text { Discomfort } \\
\text { Description }\end{array}$ & $\begin{array}{c}\text { Discomfort } \\
\text { Rating Scale }\end{array}$ \\
\hline No & 0 \\
\hline Mild & 1 \\
\hline Moderate & 2 \\
\hline Severe & 3 \\
\hline Extremely severe & 4 \\
\hline
\end{tabular}

Data was compared to the Moore Garg Strain Index (SI) of individual tasks

- SI was used to cross reference subjective impressions of the production workers

- SI assumes conducting a single task all day. 


\section{Determine Metrics}

- Part III: From the Detailed Task List, use the discomfort rating to generate a converted Ergo Points value.

- Ergonomic risk is a function of posture, repetition, force in the position of use, and duration.

- The discomfort ratings assume these variables.

- There is a non-linear relationship between the discomfort values and ergonomic risk, therefore a non-linear scale must be used to generate ergonomic risk values.

\begin{tabular}{|c|c|c|}
\hline $\begin{array}{c}\text { Discomfort } \\
\text { Description }\end{array}$ & $\begin{array}{c}\text { Discomfort } \\
\text { Rating Scale }\end{array}$ & $\begin{array}{c}\text { Ergo Points } \\
\text { Scale }\end{array}$ \\
\hline No & 0 & 3 \\
\hline Mild & 1 & 10 \\
\hline Moderate & 2 & 20 \\
\hline Severe & 3 & 50 \\
\hline $\begin{array}{c}\text { Extremely } \\
\text { severe }\end{array}$ & 4 & 100 \\
\hline
\end{tabular}




\section{Data Collection \& Analysis}

- Part IV: Determine the Process Cycle Time for each task

The Total Ergo Points values are calculated from the

\%Time/Workday for each task in a 7.5 hour workday.

$$
\begin{gathered}
\text { Total Ergo Points }= \\
\Sigma \text { Ergo Points }{ }_{(\text {Task 1-N) }} \times \text { Time }_{(\text {Task 1-N) }}
\end{gathered}
$$

- Part V: Collect 4 weeks of data

- Part VI: Conduct Statistical Analysis - in partner with UCSF 


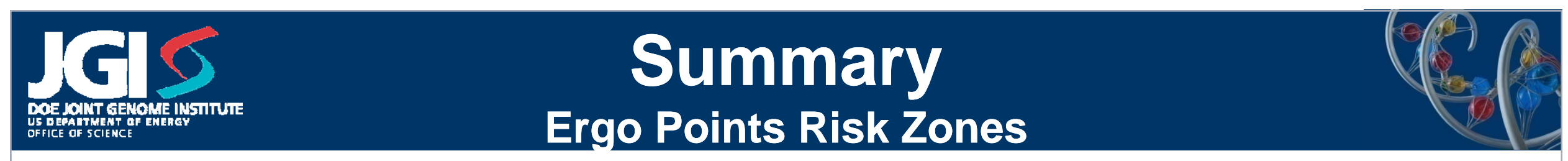

The findings of the Ergo Points Study suggest the following estimation of risk:

Ergo Points < 18 Low Risk

$18 \leq$ Ergo Points $\leq 30$ Moderate Risk

Ergo Points $>30$ High Risk
- Staff who are experiencing significant fatigue or discomfort should report this to their supervisor and should be scheduled to a task with a lower Ergo Points rating.

- Generally, schedulers should target scheduling of the staff in the Moderate Risk zone or lower.

- The Ergo Points ratings can be used to prioritize interventions. Tasks with Ergo Points ratings greater than 30 should receive a high prioritization for redesign. 


\section{Industry Tools \& Benchmarking}

- November 2005 - STEER (System to Evaluate Ergonomic Risks) forms by EORM consulting firm

- Outcome: Could not quantify overall risk for multiple tasks conducted over the course of a workday

- $\quad$ April 2006 - Moore Garg Strain Index

- Outcome: Assumes conducting only one task in a workday

- June 2006 - HAL (Hand Activity Level), NIOSH Lifting Equations, Fatigue, Liberty Mutual Manual Material Handling Tables, Static \& Dynamic Work Analysis, Rohmert Curves (muscle endurance and recovery), Rodgers Muscle Fatigue Assessment

- Outcome: Was not sensitive to high repetition multiple tasks with low hand force

- $\quad$ November 2006 - Ergo Points formulation (formerly known as Ergo Watchers)

- July 2007 - Ergo Points methodology used as guidelines for assessing risk (excel spreadsheets)

- $\quad$ March 2008 - Ergo Points software tool deployed (Kecia)

- June 2008 - Auburn Engineers eTools

- Outcome: Was not sensitive to high repetition multiple tasks with low hand force 
- Align Ergo Points and Comfort Surveys

- Transfer Local Client to Web Based Forms

- Advanced Reports

- Cross Platform Risk Assessments

- Combine Risk Assessment with Resource Allocation and Staffing Models for planning 
This work was performed under the auspices of the US Department of Energy's Office of Science, Biological and Environmental Research Program, and by the University of California, Lawrence Berkeley National Laboratory under contract No. DE-AC02-05CH11231, Lawrence Livermore National Laboratory under Contract No. DE-AC52-07NA27344, and Los Alamos National Laboratory under contract No. DE-AC02-06NA25396. 